\title{
Child Mortality Risk and Fertility: Evidence from Prevention of Mother-to-Child Transmission of HIV *
}

\author{
Nicholas Wilson \\ Department of Economics, Reed College, Portland, Oregon 97202, United States ${ }^{\dagger}$
}

First Draft: May 2011

Current Version: June 2013

\begin{abstract}
A fundamental question in development and growth is whether and how fast fertility responds to reductions in child mortality risk. I provide quasi-experimental evidence on this question from the expansion of prevention of mother-to-child transmission of HIV (PMTCT) in Zambia. My results suggest that the local introduction of PMTCT reduced pregnancy rates by approximately 10 percent, that the fertility response was greater among women who were more likely to be HIV positive, and that PMTCT substantially increased breastfeeding rates.
\end{abstract}

Keywords: child mortality risk; demographic transition; fertility; HIV/AIDS; PMTCT JEL classification: I12; J13; O12

*I would like to thank Richard Akresh, Quamrul Ashraf, Marianne Bitler, Alfredo Burlando, Raluca Buzdugan, Christopher Carpenter, Joseph Cummins, Carlos Dobkin, William Dow, Frederico Finan, Günther Fink, Jane Fortson, Andrew Foster, Paul Gertler, Erick Gong, Denise Hare, Patrick Kline, Sara Lalumia, Ronald Lee, Gianmarco Leon, Ethan Ligon, Adrienne Lucas, Jeremy Magruder, Zoë McClaren, Sandra McCoy, Edward Miguel, Nancy Padian, Gabriel Picone, Claus Pörtner, Jonathan Robinson, David Sahn, Lucie Schmidt, T. Paul Schultz, Manisha Shah, Lara Shore-Sheppard, Jeffrey Stringer, Anand Swamy, Harsha Thirumurthy, Waly Wane, Tara Watson, David Weil, Joshua Wilde, and seminar participants at the 2013 Annual Conference on Economic Development in Africa at the Centre for the Study of African Economies (CSAE) at the University of Oxford, the NBER Africa Project Zanzibar Conference, NEUDC 2012 at Yale University, the 1st NOVAFRICA Conference on Economic Development in Africa at the NOVA School of Business and Economics, the 2012 Population Association of America (PAA) Annual Meeting in San Francisco, California, the 6th Annual PopPov Conference on Population, Reproductive Health, and Economic Development in Accra, Ghana, Reed College, the University of California, Berkeley, the University of California, Irvine, the University of California, Santa Cruz, the University of Oregon, the University of South Florida, and Williams College for many excellent comments. Madeleine Watson and Wentao Xiong provided superb research assistance. The NBER Africa Project provided generous financial and institutional support. This research would not be possible without the assistance of the Network of Zambian People Living with HIV/AIDS (NZP+), the Zambia Central Statistical Office, and the Zambia Ministry of Health. All errors are my own. The findings, interpretations, and conclusions expressed in this paper are those of the author and do not necessarily represent the views of the aforementioned individuals or agencies.

${ }^{\dagger}$ Tel.: +1 Fax: +1 E-mail: nwilson@reed.edu 


\section{Introduction}

A fundamental question in development and growth is whether and how fast fertility responds to reductions in child mortality risk. As standards of living rise and public health efforts improve, poor countries have experienced large reductions in child mortality. ${ }^{1}$ Standard economic models of fertility suggest fertility may fall in response to increases in child survival and household investment in children's human capital may increase (Becker and Lewis 1973, Schultz 1997, Lee 2003). ${ }^{2}$ However, other theories of fertility do not necessarily generate this prediction and there is a classic concern that fertility may not respond to child mortality reductions, leading to rapid population growth and possibly reducing income per capita (Malthus 1798, Preston 1975, Acemoglu and Johnson 2007).

A major obstacle to answering this question is the lack of experimental evidence on the effects of child mortality risk (Schultz 1997). Many studies are purely theoretical (e.g., Becker and Lewis 1973, Sah 1991) or rely solely on non-experimental cross-sectional (or cross-country) variation in child mortality and fertility (e.g., Ben-Porath 1976, Wolpin 1984, Kalemli-Ozcan 2002, Soares 2005, Hossain et al 2007, Angeles 2010). Other studies that exploit quasi-experimental variation in disease risk (e.g., Acemoglu and Johnson 2007, Fortson 2009, Kalemli-Ozcan and Turan 2011, Juhn et al 2012, Kalemli-Ozcan 2012, Lucas 2013) are unable to isolate the effect of child mortality risk from adult mortality and morbidity risk.

I exploit the rapid expansion of a package of reproductive interventions in Zambia designed to reduce child mortality risk by reducing mother-to-child transmission of HIV ${ }^{3}$ Prevention of mother-to-child transmission of HIV (PMTCT) reduces the cumulative probability of transmission from mother-to-child by as much as 45 percent in the absence of PMTCT to as little as 3 percent (Dabis and Ekpini 2002, Canning 2006). As shown in Figure 1, between 2000 and 2007, the number of health facilities in Zambia offering PMTCT increased from fewer than 6 to nearly 600 (or 40 percent of facilities). ${ }^{4}$ This expansion should have generated large reductions in expected child

\footnotetext{
${ }^{1}$ For example, consider the two poorest regions of the world over the past twenty years. Between 1990 and 2010 , under-5 child mortality (per 1,000 live births) fell from 116 to 63 in Southern Asia and from 178 to 112 in Sub-Saharan Africa (UNICEF 2012).

${ }^{2}$ Bleakley and Lange (2009) provide evidence from hookworm eradication in the American South that the reduction in child morbidity increased household investment in children's schooling.

${ }^{3}$ One of the (if not the) main components of PMTCT is antiretroviral (ARV) prophylaxis for mothers and infants, similar to the drugs provided as antiretroviral therapy (ART) to HIV positive adults. However, the ARV regimen for PMTCT is extremely short and does not appear to have been shown to affect adult morbidity or mortality.

${ }^{4}$ This paralleled a broader scale-up of PMTCT throughout much of Sub-Saharan Africa. Between 2005 and 2008, the proportion of HIV positive pregnant women receiving PMTCT increased from 15 percent to 45 percent and the proportion of infants born to HIV positive mothers receiving PMTCT increased from 11 percent to 31 percent (WHO 2010a).
} 
mortality risk. Adult HIV prevalence in Zambia is nearly 15 percent (Central Statistical Office et al 2009). Approximately 8 percent of children in Zambia are born HIV positive or acquire HIV through breastfeeding in the absence of PMTCT (Ministry of Health 2008, Torpey et al 2010), of whom it is estimated that nearly 50 percent die by age 1 (Dabis and Ekpini 2002). ${ }^{5}$ A total fertility rate (TFR) in Zambia of 5.91 (Fortson 2009) means the representative woman in Zambia should expect to experience roughly 0.4 child deaths due to HIV over the course of her childbearing years. ${ }^{6}$

My empirical strategy is a differences-in-differences approach. I augment the Japanese International Cooperation Agency 2006 Health Facilities Census, which includes the GPS coordinates of each health facility in Zambia, with newly assembled data on the month and year each facility began offering PMTCT. Data on reproductive behavior and fertility outcomes come from repeated cross-sectional national household surveys conducted before, during, and after the scale-up, where each survey year includes respondents from multiple interview months. Combined with detailed geographic information on the location of survey households, I use these data to measure how reproductive behavior changes when PMTCT is introduced at a local health clinic. Multiple rounds of survey data before the introduction of PMTCT, as well as multiple rounds after the introduction PMTCT, mean that I am able to identify the effect of PMTCT on fertility while controlling for unobserved time-invariant characteristics of locations receiving PMTCT and differential trends between PMTCT and non-PMTCT locations.

I find evidence that the local introduction of PMTCT may have reduced pregnancy rates. My point estimates suggest that local PMTCT availability reduced pregnancy rates by approximately 2 to 4 percentage points, or roughly 10 percent relative to the mean pregnancy rate. Consistent with a causal interpretation of this main result, I find evidence suggesting that the fertility reduction was concentrated among women who were more likely to be HIV positive and among women closer to the health facility where PMTCT was locally introduced. A semi-parametric difference-indifferences analysis suggests the absence of a pre-local introduction "effect" and that the medium term response to local PMTCT introduction was larger than the short term response. I also find that local PMTCT availability increased breastfeeding rates, an important input into child quality, by between roughly 5 and 20 percentage points, or approximately 10 percent relative to the mean

\footnotetext{
${ }^{5}$ There appears to be limited evidence on the mortality-age profile for HIV positive infants who live past the age of 2 (Dabis and Ekpini 2002). Presumably in this study setting the vast majority do not survive into adolescence.

${ }^{6} \mathrm{HIV}$ /AIDS accounts for 12 percent of under-5 child mortality in Zambia (WHO 2010b). Although data do not appear to be available for mortality shares at younger ages, HIV/AIDS likely accounts for a higher fraction of under-2 mortality than under-5 mortality because roughly one-half of HIV positive infants die by age 2 (Dabis and Ekpini 2002).
} 
breastfeeding rate. $^{7}$

There are at least two important caveats about these findings. First, although pregnancy rates appear to have fallen in response to local PMTCT availability, population growth may have increased, particularly in the short term. As discussed at the end of Section 6, the fertility reduction implied by the estimated average effect of local PMTCT availability on pregnancy rates (i.e., equivalent to a 0.4 child reduction in TFR) is approximately equal to the expected child mortality reduction if PMTCT were to eliminate mother-to-child transmission of HIV. Second, we cannot rule out breastfeeding advice as the mechanism by which PMTCT reduced fertility. Although breastfeeding advice provided as part of PMTCT was intended to reduce child mortality, fertility may have fallen mechanically with lactational amenorrhea instead of women having responded to the reduction in child mortality risk by reducing fertility. However, relatively widespread knowledge of the lactational amenorrhea method (LAM) in Zambia suggests that many (although not necessarily a majority of) women were able to understand that increased breastfeeding would reduce fecundity. ${ }^{8}$

This paper contributes to the economic literature on fertility responses to changes in child mortality risk (e.g., Ben-Porath 1976, Wolpin 1984, Sah 1991, Kalemli-Ozcan 2002, Soares 2005, Hossain et al 2007, Angeles 2010) and the literature on fertility responses to changes in disease risk (e.g., Acemoglu and Johnson 2007, Fortson 2009, Lucas 2013). Similarly, the current analysis contributes to the broader literature on behavioral responses to changes in reproductive technology, including abortion (e.g., Donohue and Levitt 2001, Joyce 2004, Pop-Eleches 2006, Ananat et al 2007, Pop-Eleches 2010, Pörtner 2010, Valente 2011), contraception (e.g., Goldin and Katz 2002, Bailey 2006, Ashraf et al 2010, Miller 2010, Ananat and Hungerman 2012), and infertility treatments (e.g., Schmidt 2007, Bitler and Schmidt 2012).

This paper is among the first economic analyses of the behavioral response to PMTCT, arguably the single most effective HIV prevention intervention in existence. ${ }^{9}$ There are several economic analyses of the effects of the spread of HIV/AIDS on fertility, human capital investment, and

\footnotetext{
${ }^{7}$ This is consistent with the predictions of a quantity-quality model, in which a decrease in child quantity should be associated with an increase in child quality. However, there exist at least three alternative interpretations. First, the increase in breastfeeding could be a response to the decreased risk of infecting one's child through breastfeeding. Second, widespread use of lactational amenorrhea as a contraceptive method in the developing world means that breastfeeding may simply be the mechanism by which women are decreasing pregnancy rates. Third, PMTCT services may include recommendations to breastfeed.

${ }^{8}$ Nearly 38 percent of female respondents in the 2007 Zambia Demographic Health Survey (DHS) reported knowing of LAM (Central Statistical Office et al 2009).

${ }^{9}$ Wilson (2012) provides a primarily descriptive analysis of changes in reproductive behavior associated with the timing of local PMTCT introduction in Zambia. However, the empirical analysis in Wilson (2012) does not address omitted variables bias concerns about time specific shocks to reproductive behavior. The difference-in-differences approach in the current analysis addresses this concern by flexibly controlling for time specific shocks.
} 
economic growth (e.g., Young 2005, Young 2007, Ahuja et al 2008, Fink and Linnemayr 2009, Fortson 2009, Fortson 2011, Kalemli-Ozcan and Turan 2011, Juhn et al 2012, Kalemli-Ozcan 2012), but it is far from obvious that PMTCT should simply reverse many of these effects. One major difference between the rise of HIV/AIDS and the introduction of PMTCT is that the HIV/AIDS pandemic affects child and adult mortality, whereas PMTCT only directly affects child mortality. ${ }^{10}$

The paper is organized as follows. Section 2 describes the transmission of HIV from mother-tochild in Zambia, the medical effects of PMTCT, and the expansion of PMTCT in Zambia. Section 3 provides a conceptual framework for thinking about the behavioral response to PMTCT scale-up. Section 4 describes the household survey and health facilities census data. Section 5 explains the empirical strategy I use to identify the fertility response to PMTCT scale-up. Section 6 presents the results. Section 7 concludes.

\section{Prevention of mother-to-child transmission}

Mother-to-child transmission (MTCT) of HIV is one of the primary sources of new HIV infections. Approximately 10 percent of new HIV infections are due to MTCT (De Cock et al 2000). During the first decade of the twenty-first century, there were roughly 400,000 to 500,000 new perinatal and infant HIV infections each year (UNAIDS 2010). The vast majority of mother-to-child transmission is in Sub-Saharan Africa. More than 90 percent of pediatric HIV infections and 94 percent of pediatric AIDS deaths occur in this region (Foster and Williamson 2000).

Among pregnant women who are HIV positive, the likelihood of transmission of HIV in the absence of PMTCT is quite high. There are three ways in which a HIV positive woman may transmit the virus to her child: in utero, during childbirth, and through breastfeeding. The cumulative probability of transmission in the absence of PMTCT is between 25 and 45 percent depending on breastfeeding behavior (Dabis and Ekpini 2002). Children who acquire HIV from their mother are very likely to die before reaching age five. Between 25 and 50 percent of HIV positive infants die by age 1 . This figure rises to between 35 and 60 percent by age 2 (Spira et al 1999, Taha et al 1999, Dabis et al 2001, Brahmbhatt et al 2001). ${ }^{11}$

Prevention of mother-to-child transmission of HIV (PMTCT) generally refers to a package of

\footnotetext{
${ }^{10}$ PMTCT may indirectly affect adult mortality. In a high maternal mortality environment, changes in pregnancy rates induced by PMTCT availability should affect adult female mortality. Similarly, in a high HIV prevalence environment, changes in pregnancy-seeking behavior (i.e., unprotected sex) induced by PMTCT availability should affect adult mortality.

${ }^{11}$ There appears to be limited evidence on the mortality-age profile for HIV positive infants who live past the age of 2 (Dabis and Ekpini 2002). Presumably in this study setting the vast majority do not survive into adolescence.
} 
interventions designed to reduce mother-to-child transmission of HIV. These interventions include counseling and testing for HIV, antiretroviral drugs to reduce vertical transmission, breastfeeding advice, and family planning. However, the exact mix of interventions has changed over time with new medical evidence and increased global funding for HIV/AIDS.

In high HIV prevalence environments, PMTCT should be highly effective at reducing child mortality. Antiretroviral drugs appear to be particularly important. Combination therapy (i.e., zidovudine (ZDV) and nevirapine (NVP)) administered at the three stages at which MTCT may occur virtually eliminates MTCT (Dabis and Ekpinni 2002, Canning 2006). Single-dose nevirapine (NVP) administered at these three stages reduces MTCT by 10 to 25 percentage points (Guay et al 1999, Jackson et al 2003).

Early PMTCT efforts in Zambia appear to have focused on counseling and testing and breastfeeding advice. As early as May 2000 several clinics in Ndola city provided infant feeding guidelines and counseling and testing for prevention of mother-to-child transmission as part of routine antenatal and postnatal care (Kalibala et al 2003). Later, many clinics introduced prophylactic antiretrovirals, primarily single-dose nevirapine (NVP) initially and then combination therapy (i.e., zidovudine (ZDV) and NVP). By 2007, the National Protocol Guidelines stated an opt-out approach to HIV testing for pregnant women at antenatal clinics and codified official breastfeeding advice (Ministry of Health 2007). The 2010 National Protocol Guidelines added family planning and condom promotion as part of ongoing postnatal care (Ministry of Health 2010).

The 2007 National Protocol Guidelines (Ministry of Health 2007) appear to have been the first codified national policy on breastfeeding advice for PMTCT. The guidelines recommended that HIV negative mothers and mothers with unknown status practice exclusive breastfeeding for six months and then breastfeed up to twenty-four months. HIV positive mothers were to be "given enough information about advantages and disadvantages of the available options for them to be able to make an informed choice about what might be best for them" and advised to completely avoid breastfeeding when quality replacement feeding was available (Ministry of Health 2007). In the 2010 National Protocol Guidelines breastfeeding advice remained unchanged for HIV negative women and women with unknown status. HIV positive women were advised to practice exclusive breastfeeding for six months and then introduce complementary feeding. For HIV positive mothers with HIV positive infants, breastfeeding to twenty-four months was advised. Prior to 2007, there does not appear to have been a national breastfeeding policy for PMTCT. Evidence from Ndola city (Rutenberg et al 2003) suggests clinics may have offered advice similar to the 2007 National 
Protocol Guidelines. However, evidence from the same study suggests that breastfeeding advice varied across health workers within a given clinic. ${ }^{12}$

The expansion of antiretrovirals for PMTCT began in the early 2000s. In the capital city, Lusaka, nine antenatal clinics offered PMTCT drugs in 2002 and by January 2004 all twenty-four antenatal clinics in Lusaka city offered PMTCT drugs (Stringer et al 2005). The Center for Infectious Disease Research in Zambia (CIDRZ), in collaboration with the Elizabeth Glaeser Pediatric AIDS Foundation (EGPAF), has supported PMTCT in four of Zambia's nine provinces: Eastern, Lusaka, Southern, and Western (Elizabeth Glaser Pediatric AIDS Foundation 2011). Through the Zambia Prevention, Care, and Treatment Partnership (ZPCT), Family Health International (FHI) has supported PMTCT in the other five provinces: Northern, Luapula, Copperbelt, Central, and Northwestern (Torpey et al 2010). Single-dose nevirapine (NVP) was the main prophylaxis in the early years of the Zambian PMTCT program and zidovudine (ZDV) (also known as azidothymidine $(\mathrm{AZT})$ ) and NVP in the later years of the program (Stringer et al 2003, Stringer et al 2005, Ministry of Health 2007, Ministry of Health 2010). ${ }^{13,14}$

Figure 2 shows the expansion of PMTCT in Zambia by year of introduction. ${ }^{15}$ The figure also displays district-level population density and the main transportation routes. Higher HIV prevalence and higher population densities in urban areas mean that the average total cost of preventing mother-to-child transmission should be lower in urban areas. Consistent with this observation, PMTCT expansion was initially concentrated in the main urban areas in Zambia (e.g., Kitwe, Lusaka, and Ndola) and later grew to rural areas along the main transportation network.

The vast majority of pregnant women in Zambia visit an antenatal clinic at least once during their pregnancy, helping ensure that the scale-up of access to PMTCT translated into use of PMTCT. Among the respondents in the nationally representative survey data used in the current analysis, more than 93 percent of women who report being pregnant at any point during the twelve months prior to the survey date reported at least one visit to an antenatal clinic during that pregnancy. Perhaps surprisingly, this probability is remarkably flat across the survey rounds, varying no more than two percentage points between any two years among the four survey rounds (i.e., 2001, 2003, 2005, and 2007). Similarly, the likelihood of visiting an antenatal clinic at least once

\footnotetext{
${ }^{12}$ Goldstein et al (2012) report a similar finding at an antenatal clinic in Western Kenya.

${ }^{13}$ During the period examined in this analysis, single-dose NVP was a common prophylaxis: among HIV positive pregnant women receiving PMTCT in low-and-middle-income countries in 2007, 49 percent received single-dose NVP (WHO 2010a).

${ }^{14}$ There does not appear to be any evidence of a biochemical pathway linking these drugs to fecundity.

${ }^{15}$ In Section 4.2, I describe in more detail the data I use to construct this figure.
} 
during pregnancy is greater than 90 percent in urban areas and greater than 90 percent in rural areas. As of 2007, the modal visit to an antenatal clinic occurred during the fourth to fifth month of pregnancy (Central Statistical Office et al 2009).

Although most pregnant women visit an antenatal clinic, there are two additional possible constraints to ensuring effective PMTCT coverage. First, most births occur at home and not in a health facility. For example, in the 2007 Demographic Health Survey (DHS), 52 percent of births during the five years preceding the survey date occurred in the home (Central Statistical Office et al 2009). However, nevirapine (NVP) is provided to pregnant women upon diagnosis to be taken at home at the onset of labor and during the first few weeks of breastfeeding (Stringer et al 2003, Stringer et al 2005). Thus, home births do not appear to be a major constraint unless adherence is low among pregnant women conditional on receiving the NVP to be taken at home.

Second, evidence in Goldstein et al (2012) on the effect of health worker absence on HIV/AIDSrelated antenatal care in a clinic in Kenya suggests that health worker absence may be an important constraint in Zambia. Although there do not appear to be data on health worker absence in Zambian antenatal clinics, there is information on HIV testing during antenatal clinic visits. Among female respondents in the 2003 Zambia Sexual Behavior Survey (ZSBS) reporting an antenatal clinic visit during a pregnancy in the twelve months proceeding the survey date, 15 percent report being offered a HIV test during the visit and 45 percent of these report accepting the HIV test. By the 2005 ZSBS, these figures had increased to 27 percent and 60 percent, respectively. In the 2007 Demographic Health Survey (DHS), 64 percent of women received a HIV test during an antenatal clinic.

The rapid increase in the proportion of women receiving a HIV test at an antenatal clinic visit, combined with the relatively high acceptance rate, indicates that the physical expansion of PMTCT generated a large increase in effective coverage of HIV positive pregnant women and atrisk infants. UNICEF (2010) reports that 20 percent of HIV positive pregnant women in Zambia received PMTCT in 2004 and 8 percent of infants born to HIV positive women received PMTCT at birth. By 2007, these proportions had increased to 52 percent and 23 percent, respectively. ${ }^{16}$

\footnotetext{
${ }^{16} \mathrm{An}$ individual coverage rate that is higher than the facilities coverage rate is consistent with the higher population densities in the locations that were more likely to receive PMTCT (i.e., urban areas).
} 


\section{Conceptual framework}

\subsection{Mechanisms}

Prevention of mother-to-child transmission (PMTCT) interventions reduce the likelihood of a HIV positive woman transmitting HIV in utero, during childbirth, or to her child through breastfeeding. Antiretroviral drugs for PMTCT reduce the cumulative probability from between 25-45 percent in the absence of PMTCT to as little as 3 percent (Dabis and Ekpini 2002, Canning 2006). Between 25 and 50 percent of HIV positive children die by age 1 and between 35 to 60 percent of HIV positive children die by age 2 (Spira et al 1999, Taha et al 1999, Brahmbhatt et al 2001, Dabis et al 2001). Thus, PMTCT should increase life expectancy at birth for children born to HIV positive mothers, conditional on fertility seeking behavior and child human capital investment behavior remaining unchanged. Because PMTCT reduces child mortality risk for future (and not just current) births, local PMTCT introduction may have large effects on reproductive behavior.

A simple quantity-quality model of fertility and household investment in children's human capital (Becker and Lewis 1976) generates an ambiguous prediction about the net effect of PMTCT on fertility. The decrease in child mortality caused by PMTCT reduces the cost of child quantity. That is, PMTCT reduces the number of births the household requires to achieve a given number of surviving children. Thus, PMTCT reduces the shadow price of child quantity, leading to an increase in fertility.

Conversely, PMTCT means that infants are more likely to live on to older ages. This increases the expected return to household investments in child quality, or in other words, reduces the shadow price of child quality. This should lead to an increase in the quality of children and, under the quantity-quality tradeoff in this model, reduce the quantity of children, leading to a decrease in fertility.

Not only does PMTCT change the shadow prices of child quantity and child quality, but PMTCT also reduces the need for replacement fertility. Because PMTCT reduces child mortality, PMTCT will reduce fertility because the household will need to "replace" fewer births (i.e., births that result in child death). ${ }^{17}$ Thus, even holding the shadow prices of child quantity and child quality constant,

\footnotetext{
${ }^{17}$ Doepke (2005) examines "replacement fertility" (i.e., a mechanism where households increase fertility ex post in response to child mortality when birth choice is sequential) and "hoarding" (or precautionary fertility) (i.e., a mechanism where households increase fertility ex ante in response to expected child mortality). Doepke (2005) demonstrates that because households may accidentally have "too many" children if realized child mortality is low, replacement fertility dominates precautionary fertility as a fertility strategy in the face of child mortality. In contrast, Ben-Porath (1976) notes that hoarding (or precautionary fertility) may be important if there exists substantial risk of child mortality after a woman's childbearing years are over (e.g., if children are likely to die as adults). Because HIV
} 
we would still expect PMTCT to affect fertility.

In addition to reducing the likelihood of mother-to-child transmission, receiving PMTCT may provide information to pregnant women about their HIV status. To receive PMTCT, a pregnant woman must test HIV positive. After receiving the result of this test, she may revise her belief about her HIV status. Although evidence on the behavioral response to learning one's HIV status is mixed (Boozer and Philipson 2000, Thornton 2008, Wilson 2010, Gong 2012), the change in her beliefs may change her subsequent reproductive decisions.

\section{$3.2 \quad$ Timing}

To understand the timing of the fertility response to the local introduction of PMTCT, consider the following timeline. Let $t$ denote the month at which a woman is surveyed about her pregnancy status during the twelve months leading up to the interview date, inclusive. For women who report being pregnant at some point between $t$ and $t-11$, the likely range of possible conception dates is $t-1$ to $t-20$. Assuming individuals are perfectly informed about local PMTCT availability on the date that it is introduced, the local introduction of PTMCT by period $t-2$ or before may affect fertility behavior as measured at period $t$.

If conceptions are evenly distributed across months, then the representative pregnancy measured during the interval $t$ to $t-11$ would have begun in approximately period $t-10$. That is, the representative pregnancy would have been conceived midway through the possible conception interval, $t-1$ to $t-20$. Assuming individuals are not changing their fertility behavior in anticipation of the local introduction of PMTCT, this suggests that we are unlikely to see an effect of local PMTCT availability on pregnancy rates that are measured during the interval $t$ to $t-11$ until PMTCT has been locally available for approximately 11 months.

The lag between local PMTCT introduction and the fertility response may be even greater than this. PMTCT may affect fertility not just through decreased risk of death of future births, but also through decreased mortality among recent births. Under this latter mechanism, a decrease in pregnancy, either through lactational amenorrhea due to breastfeeding a child who is still alive or through a decrease in replacement fertility, would take longer to appear.

positive infants die with high probability within two years, whereas mortality rates among adults who acquire HIV do not begin to rise until 8-10 years after the infection date, the main risk of child mortality appears to be during the woman's childbearing years. 


\section{Data}

\subsection{Individual-level data}

Data on fertility come from the 2001 and 2007 Zambia Demographic Health Surveys (DHS) and the 2003 and 2005 Zambia Sexual Behavior Surveys (ZSBS). These are repeated cross-sectional national household surveys. Administrative records on the primary sampling units in these surveys allows me to calculate the approximate latitude and longitude of each survey household. ${ }^{18}$ This process yields 7,683 adult females (i.e., ages 15-49) in the 2001 DHS, 2,296 adult females in the 2003 ZSBS, 2,072 adult females in the 2005 ZBS, and 7,146 adult females in the 2007 DHS. ${ }^{19}$

The main fertility measure I use in this analysis is an indicator variable for whether the female respondent reported being pregnant in the twelve months leading up to the survey date. To augment the pregnancy regressions, I examine breastfeeding behavior among children age 24 months or younger.

Table 1 reports the age profiles among the DHS and ZSBS respondents for several of the key variables in this analysis. Several important facts emerge from this analysis. First, the main childbearing ages in Zambia are ages 20-29 and, to a slightly lesser extent, ages 30-39. Second, as in most high HIV prevalence countries, the HIV prevalence-age profile takes an inverted U-shape and peaks among ages 30-39. Third, the majority of children age 0-24 months breastfeed.

In Table 2, I provide mean characteristics of respondents residing in locations that ever received PMTCT and in locations that never received PMTCT. Partly because of the non-random placement of PMTCT sites, respondents in locations receiving PMTCT appear to be more educated, less likely to be married, and more likely to be HIV positive. They also have lower levels of fertility and lower breastfeeding rates for children age 0-24 months.

\footnotetext{
${ }^{18}$ The nature of the spatial information in these surveys likely introduces attenuation bias in the estimates of the fertility response to local PMTCT availability. For the ZSBS and the 2001 DHS, I use information on the respondent's Standard Enumeration Area (SEA) of residence to define the precise location of the respondent as the centroid of this SEA. These administrative units were designed to capture approximately 1,000 individuals, so although they tend to be quite small in urban areas they may be relatively large in rural areas. The 2007 DHS contains slightly different spatial information. Instead of revealing the respondent's SEA of residence, the 2007 DHS provides GPS data points that are intentionally measured with error for each respondent to address privacy concerns associated with the HIV testing module in this DHS. In the Zambia 2007 DHS, these data points were generated by adding a random vector with length drawn from a uniform distribution on 0 to 10 kilometers to the latitude and longitude of the centroid of the SEA of residence.

${ }^{19}$ The digitized census map provided by the Zambia Central Statistical Office, which I use to identify the location of the primary sampling units for the respondents in the 2001-2005 survey rounds, is missing approximately seven percent of the Statistical Enumeration Areas (SEAs) in Zambia. Thus, I am unable to identify the precise location of approximately seven percent of the 2001-2005 survey respondents and exclude these respondents from the empirical analysis.
} 


\subsection{Health facilities data}

We collected retrospective data on the month and year each health facility began offering each of the three main HIV/AIDS services: PMTCT, antiretroviral therapy (ART), and voluntary counseling and testing (VCT). The 2006 Japanese International Cooperation Agency (JICA) Health Facility Census (HFC) surveyed each health facility in Zambia and recorded the exact latitude and longitude of each health site. To augment these data, we re-surveyed these clinics to collect information on the month and year (if any) they began offering each of the three main HIV/AIDS services. This process effectively began in June of 2008 so this retrospective panel provides comprehensive information on the expansion of HIV/AIDS services in Zambia through the middle of $2008 .^{20,21}$

\section{$5 \quad$ Empirical strategy}

The main empirical strategy in this paper is to measure the change in fertility associated with the local introduction of PMTCT while controlling for a host of time and geographic fixed effects and linear trends. To implement this strategy, I use the detailed spatial information in the JICA 2006 HFC and the household survey data to calculate the distance from each survey respondent to each health facility and measure fertility among respondents who reside near health facilities currently offering PMTCT. Because I have information on fertility in locations receiving PMTCT before they received PMTCT, I am able to control for unobservable characteristics (both time-invariant and time-varying) affecting fertility that are associated with the placement of PMTCT sites. PMTCT was not introduced everywhere simultaneously, so instead of including a "post" indicator as in a standard difference-in-differences specification I include survey month times survey year fixed effects. This controls for unobserved time-specific shocks to fertility. I also include province fixed

\footnotetext{
${ }^{20}$ Service interruptions (e.g., because of ARV shortages or health worker absence) mean that PMTCT may not have been continuously available at all of these clinics from the local introduction date onward. However, this should only work against the regression analysis yielding any estimate effect of local PMTCT availability on pregnancy rates. The fact that I find a large and statistically significant effect suggests that the behavioral response to any service interruptions was not large enough to outweigh the effect of the documented service availability.

${ }^{21}$ Existing national level data from other sources do not offer a precise account of aggregate PMTCT expansion in Zambia. The Ministry of Health and the National AIDS Council (2008) state, "Overall, PMTCT services have been rolled out to all the 72 districts of Zambia, representing an increase from 67 in 2005, 307 in 2006 , and 678 as of September 2007. This scaling up of PMTCT services resulted in an increase in pregnant women who completed prophylaxis from 14,071 in 2005 to 25,578 in 2006, and by September 2007 this figure had reached 35,314." In contrast, the National AIDS Council (2006) reports that there were 270 PMTCT sites in Zambia by mid-2005. PEPFAR reports supporting PMTCT services in Zambia at 95 clinics in fiscal year 2004 (PEPFAR 2005), 200 clinics in Zambia in fiscal year 2005 (PEPFAR 2006), and 284 clinics in fiscal year 2006 (PEPFAR 2007). Bweupe (2006) reports other data from the Ministry of Health indicating that the number of PMTCT sites in Zambia increased from 6 in 2001, to 64 in 2003, to 265 sites in 2005. Using data from the UNICEF country office, Ngashi et al (2006) reports that the number of PMTCT sites in Zambia increased from 80 to 254 in a single year.
} 
effects to control for additional time-invariant spatial heterogeneity. ${ }^{22}$

There remain at least four potential threats to identifying the causal effect of local PMTCT availability on fertility. First, PMTCT may have been introduced in locations that were following a different time trend than those locations that did not receive PMTCT. ${ }^{23}$ To address this concern, I include province-specific linear time trends and a linear time trend for locations ever receiving PMTCT. Second, the expansion of ART and VCT may have been correlated with the expansion of PMTCT. Thus, I use information from the augmented-HFC to control for proximity to ART and VCT. ${ }^{24}$ Third, the expansion of PMTCT may have been correlated with the expansion of two important inputs into child health, household access to piped water and household bed net ownership. I address this concern by controlling for household access to piped water and household bed net ownership as reported in the DHS and ZSBS. ${ }^{25}$ Fourth, locations receiving PMTCT early may have had fundamentally different fertility behavior than those locations receiving PMTCT later. This is a concern because respondents in locations ultimately receiving PMTCT but where PMTCT was not available around the time of conception are a control group for early PMTCT recipients. Thus, I include an indicator variable equal to one if the respondent resides in a location that was a late PMTCT recipient. ${ }^{26}$

Thus, the primary regression equation is:

$$
\begin{aligned}
\text { fertility }_{i j m t}= & \alpha_{1} \text { PMTCT }_{i j(m t-11)}+\alpha_{2} \text { PMTCTever }_{i j}+\alpha_{3} \text { PMTCTlate }_{i j}+X_{i j m t}^{\prime} \Gamma \\
& +\eta_{j}+\delta_{m t}+m t \times \mu_{j}+m t \times \text { PMTCTever }_{i j}+\epsilon_{i j m t}
\end{aligned}
$$

where fertility ijm $_{\text {t }}$ denotes the reproductive behavior of female respondent $i$ residing in province $j$ in month $m$ in year $t, P M T C T_{i j(m t-11)}$ is an indicator variable equal to one if a health clinic offering PMTCT at least 11 months prior to the survey date is located near respondent $i$, PMTCTever $_{i j}$ is an indicator variable equal to one if a health clinic located near respondent $i$ ever offered PMTCT even if it was subsequent to the interview date for respondent $i$, PMTCTlate $_{i j}$ is an indicator vari-

\footnotetext{
${ }^{22}$ This is a concern because I do not observe fertility before and after local PMTCT introduction in every location where I have household survey respondents. There are nine provinces in Zambia.

${ }^{23}$ The rural-urban difference in fertility trends during the period spanned by the household survey data support this hypothesis. Between 2001 and 2007, rural fertility increased from 6.9 births per woman to 7.5 births per woman, whereas urban fertility remained constant at 4.3 births per woman (Central Statistical Office et al 2009).

${ }^{24}$ In the specifications in which I control for ART and VCT availability, I include the ART (and VCT) analogues of $P M T C T_{i j(m t-11)}$ and PMTCTever $i j$.

${ }^{25}$ The 2003 ZSBS does not include information on household bed net ownership.

${ }^{26}$ I define a location as a late PMTCT recipient if it received PMTCT after December 2004.
} 
able for late PMTCT recipient, $X_{i j t}$ is a vector of individual and household level demographic controls (i.e., indicator variables for five year age group, primary school completion, secondary school completion, marital status, local ART availability, local VCT availability, household bed net ownership, and piped water access), $\eta_{j}$ are province fixed effects, $\delta_{m t}$ are survey month times survey year fixed effects, $m t \times \mu_{j}$ are province-specific linear trends, and $m t \times P M T C$ Tever $_{i j}$ is a linear trend for locations ever receiving PMTCT. ${ }^{27}$ I estimate the parameters of Equation (1) in a linear probability model (i.e., using ordinary least squares (OLS) regression). As in a standard differencein-differences empirical strategy, I interpret $\alpha_{1}$ as the causal effect of local PMTCT availability on fertility. ${ }^{28}$

I measure fertility using an indicator variable equal to one if the respondent reported being pregnant at some point during the twelve months prior to the survey month, inclusive. During the period I examine in this analysis, the representative location with PMTCT in Zambia received it around 2005. Birth spacing in Zambia is roughly 3 to 4 years. Thus, the representative woman exposed to PMTCT in Zambia only faced one (or fewer) likely births after the local introduction of PMTCT. Because very few women were at risk of having more than one birth during this period, I focus on pregnancy rates as my primary measure of fertility behavior. I also examine breastfeeding behavior among a sample of children age 0-24 months in a child level regression analogous to Equation (1) with mother's demographic characteristics as the individual level controls, as well as an indicator variable for child age 13-24 months.

The baseline specification defines a respondent as being near a health clinic if the respondent lives within 20 kilometers of the nearest health clinic. In an analysis of the correlates of maternal health care usage in Kalabo District in Zambia, Stekelenburg et al (2004) find that usage rates decline substantially for women residing more than a two-hour walk from a maternal health care site. Similarly, female respondents in the 2007 DHS indicate that distance is one of the primary barriers to seeking health care (Central Statistical Office et al 2009). In alternative specifications I relax the restriction that the local introduction of PMTCT has the same effect on behavior invariant of distance conditional on whether distance was greater or less than 20 kilometers.

Standard errors are clustered at the Statistical Enumeration Area (SEA) level. My distance based measure of PMTCT availability varies at the level of the SEA. As indicated in Cameron et

\footnotetext{
${ }^{27}$ In the breastfeeding regressions, the individual level controls also include an indicator variable for child age 13-24 months.

${ }^{28}$ In a difference-in-differences interpretation of this regression specification, PMTCTij(mt - 11) is "treatment" interacted with "post", PMTCTever ${ }_{i j}$ is the "treatment" indicator variable, and the survey month times survey year fixed effects correspond to the standard "post" variable.
} 
al (2008), the more than 300 SEAs in the 2001 DHS and more than 300 SEAs in the 2007 DHS mean that standard asymptotic tests are appropriate.

\section{Results}

\subsection{Effect of local PMTCT on pregnancy and breastfeeding}

The main regression results suggest that the local introduction of PMTCT may have reduced pregnancy and increased breastfeeding rates. Estimates of the effect of local PMTCT availability on pregnancy and breastfeeding appear in Panels A and B, respectively, of Table 3. All specifications include an indicator variable equal to one if a clinic within 20 kilometers of the respondent ever offered PMTCT. Standard errors are clustered by Standard Enumeration Area (SEA).

Panel A examines the effect of local PMTCT availability on the likelihood the respondent was pregnant at any point in the twelve months preceding the survey date. In Column (1), I present the results of a simple specification that only controls for whether a health facility within 20 kilometers of the respondent ever offered PMTCT. The point estimate indicates that local PMTCT availability (i.e., at a clinic located within 20 kilometers of the respondent) reduced the likelihood that the respondent was pregnant by 4.6 percentage points. This effect is fairly large not only in absolute terms, but also in relative terms and is statistically significant at the 1 percent level. Compared to the mean pregnant rate of 0.31 in the entire sample, a 4.6 percentage point reduction corresponds to approximately a 15 percent reduction in pregnancy rates. ${ }^{29}$

In general, the estimated effect of local PMTCT availability on pregnancy is relatively robust to including additional controls. The main exception to this pattern is in Column (6), where the point estimate falls to -0.020 and is no longer statistically significant at conventional levels (pvalue $=0.241$. One interpretation of this finding is that local PMTCT introduction did not generate a statistically significant effect on pregnancy rates on average in the full sample. ${ }^{30}$ An alternative interpretation is that it is difficult to separately identify the effects of local PMTCT from other

\footnotetext{
${ }^{29}$ An alternative explanation for the effect of local PMTCT availability on pregnancy is that PMTCT may be bundled with additional emphasis on family planning services. However, empirical evidence does not support this hypothesis. The 2001 and 2007 DHS ask respondents whether a health worker discussed family planning with the respondent during a visit during a pregnancy in the twelve months leading up to the survey date. Using the same specification as in Column (6) of Table 3, OLS regression results suggest that local PMTCT introduction did not generate a substantial increase in the likelihood a health worker discussed family planning with the respondent. The coefficient on local PMTCT availability is virtually zero (i.e., -0.00016, p-value=0.997.)

${ }^{30}$ As discussed in Sections 6.3 and 6.4, the regression estimates suggest statistically significant reductions in pregnancy rates in the medium term (i.e., at least 36 months after local introduction) and among respondents more likely to be HIV positive.
} 
HIV/AIDS services and two other important inputs (i.e., piped water and bed nets) affecting child health.

Panel B examines the effect of local PMTCT availability on breastfeeding. Among HIV positive women in the absence of PMTCT, avoiding breastfeeding reduces the probability of mother-tochild transmission (MTCT) by roughly 15-20 percentage points (Newell et al 2004). However, breastfeeding is an important nutritional input for infants, particularly in resource-constrained settings. Thus, current World Health Organization (WHO) guidelines in resource-poor settings recommend breastfeeding for women who have unknown HIV status and even for women who are known to be HIV positive (WHO 2009). Data on breastfeeding come from the 2001 and 2007 DHS survey years. These regressions use the child age 0-24 months as the unit of observation instead of the mother and restrict the sample of children to those born January 1999 or later. ${ }^{31}$

Throughout, the estimates presented in Panel B suggest that the local introduction of PMTCT substantially increased breastfeeding. ${ }^{32}$ For example, in Column (1), local PMTCT availability is associated with a 3.7 percentage point increase in the likelihood of breastfeeding, an estimate that is statistically significant at the 5 percent level. The estimated positive effect of local PMTCT availability is highly robust to including additional controls. However, the magnitude of the effect is quite sensitive to which controls are included. For example, in the specification with the full set of controls (i.e., Column (6)), the estimated effect of local PMTCT availability is 23.6 percentage points (significant at the 1 percent level).

\subsection{Spatial heterogeneity}

The results in Table 3 suggest that the local introduction of PMTCT reduced pregnancy rates and increased breastfeeding rates. If this is true, then we would expect to see larger effects closer to the location where PMTCT was introduced and smaller effects in the periphery of this location. Table 4 explores this question by allowing the effect of local PMTCT introduction to vary by the respondent's distance to the location where PMTCT was introduced.

Panel A examines spatial heterogeneity in the effect of local PMTCT introduction on pregnancy

\footnotetext{
${ }^{31}$ The median breastfeeding duration in Zambia is approximately 20 months (Central Statistical Office et al 2009).

${ }^{32} \mathrm{In}$ an analysis of the effect of health worker absence at a single antenatal clinic in Western Kenya on health outcomes, Goldstein et al (2012) find that the presence of a PMTCT nurse when a pregnant woman visits an antenatal clinic is associated with no change in the probability of receiving PMTCT or in the probability of breastfeeding. However, among the subset of pregnant women who believe they are HIV positive the presence of a PMTCT nurse increases the probability they receive PMTCT by 7.4 percentage points and decreases the probability they breastfeed by 9 percentage points. The seemingly divergent effects documented in Goldstein et al (2012) and in the current analysis suggest that there is heterogeneity across locations in the advice and care that antenatal clinics provide clients.
} 
rates. The point estimates suggest that the reduction in pregnancy rates was greater for individuals residing within 10 kilometers of local introduction than for individuals residing at greater distances. To fix ideas, consider the specification with the full set of controls (i.e., Column (6)). These estimates suggest that PMTCT introduction within 10 kilometers reduced the likelihood of being pregnant by 2.7 percentage points (for F-test of joint significance, p-value=0.153). PMTCT introduction within 11-20 kilometers was only weakly associated with a 0.6 percentage point reduction (statistically insignificant at conventional levels) and PMTCT introduction within 21-30 kilometers was actually associated with a 0.7 percentage point increase (albeit statistically insignificant) in the likelihood of pregnancy.

Panel B repeats this exercise for the breastfeeding regressions. In contrast to the pregnancy regressions, PMTCT availability appears to have increased breastfeeding rates equally among respondents residing within 10 kilometers and those residing 11-20 kilometers from local introduction. Similar to the pregnancy regressions, PMTCT availability appears to have had no effect on breastfeeding among respondents residing 21-30 kilometers from local introduction.

\subsection{Effect dynamics}

The longitudinal dimension of these data enables several additional analyses that support a causal interpretation of the baseline results. Figure 3 provides a semi-parametric difference-in-differences analysis of the effect of local PMTCT availability on pregnancy. ${ }^{33}$ This figure plots the estimates of $\beta_{k}$ from the following regression equation:

$$
\begin{aligned}
\text { fertility }_{i j m t}= & \sum_{k=-96}^{84} \beta_{k} 1\left(\tau_{i j m t}=k\right)+\gamma_{1} \text { PMTCTever }_{i j}+\gamma_{2} \text { PMTCTlate }_{i j}+X_{i j m t}^{\prime} \Gamma \\
& +\eta_{j}+\delta_{m t}+m t \times \mu_{j}+m t \times \text { PMTCTever }_{i j}+\epsilon_{i j m t}
\end{aligned}
$$

where $\tau_{\text {ijmt }}$ denotes the twelve (or eleven) month event window and is defined such that $\tau=0$ for respondents surveyed 0 to 10 months after the local introduction of PMTCT, $\tau=1$ for respondents surveyed 11 to 23 months after the local introduction of PMTCT, $\tau=2$ for respondents surveyed 24 to 35 months after the local introduction of PMTCT, and so forth. For $\tau<0$, respondents were

\footnotetext{
${ }^{33}$ Unlike an event study, I include "untreated" respondents (i.e., individuals residing further than 20 kilometers from an eventual PMTCT site) in these regressions. A pure event study analysis that omits these respondents yields qualitatively similar results: little evidence of a pre-introduction trend and a clear downward trend beginning shortly after local introduction.
} 
surveyed prior to the local introduction of PMTCT. Aside from the event study parameters, this specification is identical to the specification in Equation (1). As with Equation (1), I estimate the parameters of Equation (2) in a linear probability model (i.e., using ordinary least squares (OLS) regression). Figure 3 also reports the results from estimating the quasi-event study parameters of a version of Equation (2) that includes no controls.

The coefficient estimates plotted in Figure 3 are broadly consistent with a causal interpretation of the baseline pregnancy results. There appears to have been a reduction in pregnancy rates in locations receiving PMTCT six to eight years prior to local PMTCT introduction. However, during the five years prior to local PMTCT introduction, there is no evidence a pre-local introduction trend in pregnancy in locations ultimately receiving PMTCT. Furthermore, there is evidence of a clear downward trend in the point estimates that begins shortly after the local introduction of PMTCT. ${ }^{34}$

Table 5 continues this dynamic analysis by estimating a version of Equation (1) that allows the fertility response to vary by the length of time it has been available. In particular, I allow for an additional effect of PMTCT on fertility in locations in which PMTCT was available for at least thirty-six months. The coefficient estimates in Panel A suggest that although pregnancy may have responded in the short term, it was only after PMTCT had been available for at least thirty-six months that the full sample of respondents demonstrated a statistically significant and robust reduction in the likelihood of pregnancy. ${ }^{35}$ Moreover, the point estimates suggest that the medium term response was approximately twice as large as the short term response. In contrast, the coefficient estimates in Panel B suggest that the breastfeeding response may have been insensitive to the duration PMTCT was locally available.

\subsection{Heterogeneity by HIV prevalence}

Presumably, women who were more likely to have been HIV positive should have demonstrated a greater fertility response to the local availability of PMTCT. Because the 2007 DHS is the only survey round that includes information on HIV status that is linked to information on fertility decisions, I cannot examine how the response varied by the respondents' actual HIV status. However, I can examine how it varied by HIV prevalence across demographic group as defined by the

\footnotetext{
${ }^{34}$ In Figure 3, "0" on the X-axis denotes 0-11 months after local PMTCT introduction. "12" on the X-axis denotes 12-23 months after local PMTCT introduction and corresponds to the first period I include in the main measure of PMTCT availability in main regression specification (i.e., Equation (1)).

${ }^{35}$ For example, the point estimates in Column (6) in Table 5 suggest a 3.6 percentage point reduction (statistically significant at the 10 percent level) in pregnancy rates in locations where PMTCT had been available at least thirty-six months.
} 
interaction of gender, five-year age group, and province of residence. ${ }^{36}$

Table 6 presents the results of pregnancy regressions that allow the fertility response to vary by HIV prevalence among the respondent's demographic group. Because HIV prevalence is defined using geographic and demographic characteristics, caution should be used in interpreting results from specifications that do not include these controls (i.e., Columns (1)-(4)). Panel A uses the continuous measure of HIV prevalence in the respondent's demographic group. Although the sign on the HIV prevalence interaction term is negative, it is very imprecisely estimated and usually is not statistically significant. Panel B uses an indicator variable equal to one if the respondent was in a demographic group with HIV prevalence at or above the median level. In these regressions, the estimated fertility response appears to have been twice as large among women in high HIV prevalence groups, consistent with a causal interpretation of the estimated effects.

Table 7 reports the analogous breastfeeding regressions. As in Table 6, I explore two specifications. Throughout, there is little evidence that the breastfeeding response was larger among women who were more likely to be HIV positive. In fact, the coefficient estimate on the HIV prevalence interaction term tends to be close to zero, although it is imprecisely estimated.

\subsection{Discussion}

These results suggest three main inferences about the fertility response to local PMTCT availability. First, local PMTCT introduction appears to have reduced pregnancy rates. Whether this reflects a reduction in the desired number of children due to a quantity-quality shift or is simply part of a reduction in replacement fertility is unclear. ${ }^{37}$ However, a simple calculation suggests we cannot rule out a pure replacement fertility explanation. The representative woman in Zambia should expect to experience roughly 0.4 child deaths due to HIV over the course of her childbearing years. $^{38}$ If PMTCT expansion is expected to eliminate these deaths, then there should be a 0.4

\footnotetext{
${ }^{36}$ There is substantial variation in HIV prevalence across age groups and provinces. For example, as shown in Table 2, HIV prevalence is approximately four times as high among women age 30-34 and 35-39 than among women age 15-19. Similarly, HIV prevalence in Lusaka Province is nearly three times as high as in Northern Province. In addition, aggregate HIV prevalence in Zambia has been relatively flat during the period 2001-07 (Central Statistical Office et al 2009).

${ }^{37} \mathrm{~A}$ larger estimated response in the medium term than in the short term provides some support for the replacement fertility hypothesis. However, this dynamic effect is also consistent with social learning about PMTCT availability or a relaxation of supply side constraints (e.g., interruptions in antiretroviral supply possibly associated with early expansion).

${ }^{38}$ Of the roughly 8 percent of children born HIV positive or who acquire HIV through breastfeeding (Ministry of Health 2008, Torpey et al 2010), nearly one-half die by age 1 (Dabis and Ekpini 2002). Given a total fertility rate (TFR) of 5.91 in Zambia (Fortson 2009), the representative woman should expect to experience approximately 0.4 child deaths due to HIV over the course of her childbearing years.
} 
child reduction in number of expected child deaths. A 0.4 child (or 6.76 percent) reduction in TFR would compensate for this reduction and leave the number of surviving children unchanged. The baseline estimate from the specification with the full set of controls (i.e., Column (6) of Table 3, Panel A) is a 2.1 percentage point (or 6.76 percent) reduction in pregnancy rates, consistent with no change in the quantity of surviving children. On the other hand, the estimated change in locations where PMTCT has been available at least 36 months was a 4 percentage point (or approximately 13 percent) reduction in pregnancy rates. A 13 percent reduction in TFR is a reduction of approximately 0.76 children, or nearly twice the expected reduction in child mortality.

Second, local PMTCT availability appears to have increased breastfeeding rates. Again, these data are not rich enough to fully adjudicate between four competing hypotheses: (i) breastfeeding increased because breastfeeding (i.e., the lactational amenorrhea method (LAM)) is an important contraceptive method in Zambia ${ }^{39}$ and women increased breastfeeding to reduce fertility, (ii) breastfeeding increased because of a quantity-quality shift associated with the reduction in the likelihood of child death, (iii) breastfeeding increased because the risk of transmission through breastfeeding fell, or (iv) breastfeeding increased because of breastfeeding advice provided as part of PMTCT. The lack of a differential breastfeeding response by the likelihood the mother was HIV positive suggests that breastfeeding advice may be the mechanism by which PMTCT affected breastfeeding. There is some evidence that PMTCT sites may have recommended breastfeeding for HIV positive mothers and HIV negative mothers alike (Ministry of Health 2010). However, detailed data on breastfeeding advice during this period in Zambia are not available and breastfeeding advice was only formalized at the national level in the 2007 National Protocol Guidelines (i.e., after the period during which the effect of PMTCT on fertility is measured in the current analysis).

Third, the overall fertility response may have been due to the reduction in child mortality risk associated with local PMTCT introduction. All components of PMTCT (e.g., HIV testing, antiretroviral drugs, family planning) are designed to reduce child mortality risk due to MTCT. If women were informed about the basic reasoning behind the behavioral change components (e.g., breastfeeding advice) then it is more plausible that the observed fertility change was a response to the reduction in child mortality risk. However, if the observed change in fertility was due to an expansion in contraception supply that affected behavior independent of HIV status, then it becomes more difficult to interpret these results as the fertility response to reductions in child

\footnotetext{
${ }^{39}$ Sixteen percent of all adult women have ever used LAM and twenty-two percent of married women have ever used LAM (Central Statistical Office 2009).
} 
mortality risk.

\section{Conclusion}

This paper examines fertility responses to a dramatic change in the availability of a critical reproductive technology, prevention of mother-to-child transmission of HIV (PMTCT), in one of the highest HIV prevalence and highest fertility countries in the world. The quasi-experimental evidence I present suggests that PMTCT scale-up in Zambia generated a reduction in pregnancy rates for respondents residing near health facilities offering PMTCT. ${ }^{40}$ My estimates suggest that local PMTCT availability (i.e., at a clinic within 20 kilometers of a respondent) reduced the likelihood the respondent was pregnant by approximately 2 to 4 percentage points (i.e., roughly a 10 percent reduction). Larger estimated responses among women who were more likely to be HIV positive, closer to the facility where PMTCT was locally introduced, and in the medium term than in the short term support a causal interpretation of this estimated effect. In addition to these findings, the results suggest that local PMTCT availability increased breastfeeding rates by approximately 10 percent, indicating that PMTCT may have generated increased investment in child quality.

These results suggest that the continued expansion of PMTCT in Sub-Saharan Africa may reduce pregnancy rates, as well as increase investments in child quality at a critical age. Because women and households likely will spend fewer resources in the pursuit of child quantity, this may release resources (including female labor) previously allocated to the production of child quantity for alternative uses. This suggests that PMTCT scale-up is not just a humanitarian success, but may also promote economic development and growth by accelerating the demographic transition in high HIV prevalence, high fertility countries. Future research should examine the long-term fertility response to PMTCT.

\footnotetext{
${ }^{40}$ Notably, the estimated effect of local PMTCT availability in the baseline specification with the full set of controls (i.e., Column (6) of Table 3) is not statistically significant at conventional levels. However, the regression specifications that allow for heterogeneous effects reveal statistically significant reductions in the medium term (i.e., after PMTCT had been locally available at least thirty-six months) and among women who more likely to be HIV positive.
} 


\section{References}

Acemoglu, Daron and Simon Johnson. 2007. Disease and development: The effect of life expectancy on economic growth. Journal of Political Economy, 115(6): 925-985.

Ahuja, Amrita, Brian Wendell, and Eric Werker. 2008. Male circumcision and AIDS: The macroeconomic impact of a health crisis. Unpublished manuscript.

Ananat, Elizabeth Oltmans, Jonathon Gruber, and Phillip Levine. 2007. Abortion legalization and life-cycle fertility. Journal of Human Resources, 42: 375-397

Ananat, Elizabeth Oltmans and Daniel Hungerman. 2012. The power of the pill for the next generation. Review of Economics and Statistics, forthcoming.

Angeles, Luis. 2010. Demographic transitions: Analyzing the effects of mortality on fertility. Journal of Population Economics, 23: 99-120.

Ashraf, Nava, Erica Field, and Jean Lee. 2010. Household bargaining and excess fertility: An experimental study in Zambia. BREAD Working Paper No. 282.

Bailey, Martha. 2006. More power to the pill: The impact of contraceptive freedom on women's lifecycle labor supply. Quarterly Journal of Economics, 121(289).

Becker, Gary and H. Gregg Lewis. 1973. On the interaction between the quantity and quality of children. Journal of Political Economy, 81(2, Part 2): S279-S288.

Ben-Porath, Yoram. 1976. Fertility response to child mortality: Micro data from Israel. Journal of Political Economy, 84(4) Part 2: S163-S178.

Bleakley, Hoyt and Fabian Lange. 2009. Chronic disease burden and the interaction of education, fertility, and growth. Review of Economics and Statistics, 91(1): 52-65.

Bitler, Marianne and Lucie Schmidt. 2012. Utilization of infertility treatments: The effects of insurance mandates. Demography, 49(1), 124-149.

Boozer, Michael, Philipson, Tomas. 2000. The impact of public testing for Human Immunodeciency Virus. The Journal of Human Resources, 35(3), 419-446.

Brahmbhatt, Heena, Fred Wabwire-Mangen, Godfrey Kigozi, Ronald Gray. 2001. Associations of maternal HIV and child survival in Rakai, Uganda. 3rd Conference on global strategies for the prevention of HIV transmission from mothers to infants. September 9-13, 2001, Kampala, Uganda (abstract 59).

Bweupe, Max. 2006. Successful scaling up approaches to PMTCT in resource limited settings: The Zambian experience. The XVI International AIDS Conference, Toronto, Canada.

Cameron, A. Colin, Jonah Gelbach, and Douglas Miller. 2008. Bootstrap-based improvements for inference with clustered errors. Review of Economics and Statistics, 90(3): 414-427.

Canning, David. 2006. The economics of HIV/AIDS in low-income countries: The case for prevention. Journal of Economic Perspectives, 20(3): 121-142.

Central Statistical Office, Ministry of Health, Tropical Diseases Research Centre, University of Zam- 
bia, and Macro International Inc. 2009. Zambia Demographic and Health Survey 2007. Calverton, Maryland, USA: CSO and Macro International inc.

Dabis, Francois, Narcisse Elenga, Nicolasc Meda, Valeriane Leroy, Ida Viho, Olivier Manigart, Laurence Dequae-Merchadou, Phillipe Msellati, Issiaka Sombie. 2001. 18-month mortality and perinatal exposure to Zidovudine in West Africa. AIDS, 15: 771-779.

Dabis, Francois and Ehounou Rene Ekpini. 2002. HIV-1/AIDS and maternal and child health in Africa. Lancet, 359: 2097-2104.

De Cock, Kevin, Mary Glenn Fowler, Eric Mercier, Isabelle de Vincenzi, Joseph Saba, Elizabeth Hoff, David Alnwick, Martha Rogers, and Nathan Shaffer. 2000. Prevention of mother-to-child HIV transmission in resource-poor countries: Translating research into policy and practice. Journal of the American Medical Association, 283(9): 1175-1182.

Doepke, Matthias. 2005. Child mortality and fertility decline: Does the Barro-Becker model fit the facts? Journal of Population Economics, 18: 337-366.

Donohue, John and Stephen Levitt. 2001. The impact of legalized abortion on crime. Quarterly Journal of Economics, 116(2): 379-420.

Elizabeth Glaser Pediatric AIDS Foundation. 2011. www.pedaids.org

Fink, Günther and Sebastian Linnemayr. 2009. HIV does matter for fertility: Human capital, mortality, and family size. Harvard University working paper.

Fortson, Jane. 2009. HIV/AIDS and fertility. American Economic Journal: Applied Economics, 1(3): 170-194.

Fortson, Jane. 2011. Mortality risk and human capital investment: The impact of HIV/AIDS in Sub-Saharan Africa. Review of Economics and Statistics, 93(1): 1-15.

Foster, Geoff and John Williamson. 2000. A review of current literature of the impact of HIV/AIDS on children in Sub-Saharan Africa. AIDS, 14(supplement 3): S275-S284.

Goldin, Claudia and Lawrence Katz. 2002. The power of the pill: Oral contraceptives and women's career and marriage decisions. Journal of Political Economy, 110(4): 730-770.

Goldstein, Markus, Joshua Graff Zivin, James Habyarimana, Cristian Pop-Eleches, and Harsha Thirumurthy. 2012. The effect of health worker absence and health clinic protocol on health outcomes: the case of mother-to-child transmission of HIV in Kenya. American Economic Journal: Applied Economics, forthcoming.

Gong, Erick. 2012. HIV testing and risky sexual behavior. Middlebury College working paper.

Guay, Laura, Philippa Musoke, Thomas Fleming, Danstan Bagenda, Melissa Allen, Clemensia Nakabiito, Joseph Sherman, Paul Bakaki, Constance Ducar, Martina Deseyve, Lynda Emel, Mark Mirochnick, Mary Glenn Fowler, Lynne Mofenson, Paolo Miotti, Kevin Dransfeld, Dorothy Bray, Francis Mmiro, and J. Brooks Jackson. 1999. Intrapartum and neonatal single-dose nevirapine compared with zidovudine for prevention of mother-to-child transmission of HIV-1 in Kampala, Uganda: HIVNET 012 randomised trial. The Lancet, 354(September 4): 795-802.

Hossain, Mian Bazle, James Phillips, and Thomas Legrand. 2007. The impact of childhood mor- 
tality on fertility in six rural thanas of Bangladesh. Demography, 44(4): 771-784.

Jackson, J. Brooks, Philippa Musoke, Thomas Fleming, Laura A Guay, Danstan Bagenda, Melissa Allen, Clemensia Nakabiito, Joseph Sherman, Paul Bakaki, Maxensia Owor, Constance Ducar, Martina Deseyve, Anthony Mwatha, Lynda Emel, Corey Duefeld, Mark Mirochnick, Mary Glenn Fowler, Lynne Mofenson, Paolo Miotti, Maria Gigliotti, Dorothy Bray, Francis Mmiro. 2003. Intrapartum and neonatal single-dose nevirapine compared with zidovudine for prevention of motherto-child transmission of HIV-1 in Kampala, Uganda: 18-month follow-up of the HIVNET 012 randomised trial. The Lancet, 362(September 13): 859-868.

Joyce, Ted. 2004. Did legalized abortion lower crime? Journal of Human Resources, 39(December): $1-28$.

Juhn, Chinhui, Sebnem Kalemi-Ozcan, Belgi Turan. 2012. HIV and fertility in Africa: First evidence from population-based surveys. Journal of Population Economics, forthcoming.

Kalibala, S. et al 2003. Empowering Communities to Respond to HIV/AIDS: Ndola Demonstration Project on Maternal and Child Health. Population Council: Washington, DC.

Kalemli-Ozcan, Sebnem. 2002. Does the mortality decline promote economic growth? Journal of Economic Growth, 7: 411-439.

Kalemli-Ozcan, Sebnem. 2012. AIDS, "reversal" of the demographic transition and economic development: Evidence from Africa. Journal of Population Economics, 25(3): 871-897.

Kalemli-Ozcan, Sebnem and Belgi Turan. 2011. HIV and fertility revisited. Journal of Development Economics, 96(1): 6165 .

Lee, Ronald. 2003. The demographic transition: Three centuries of fundamental change. The Journal of Economic Perspectives, 17(4): 167-190.

Lucas, Adrienne. 2013. The impact of malaria eradication on fertility. Economic Development and Cultural Change, forthcoming.

Malthus, Thomas. 1798. An Essay on the Principle of Population. London: J. Johnson, St. Paul's Churchyard.

Miller, Grant. 2010. Contraception as development? New evidence from family planning in Colombia. Economic Journal, 120(545): 709-736.

Ministry of Health, Zambia. 2007. PMTCT National Protocol Guidelines. Lusaka, Zambia.

Ministry of Health, Zambia. 2008. PMTCT National Protocol Guidelines. Lusaka, Zambia.

Ministry of Health, Zambia. 2010. PMTCT National Protocol Guidelines. Lusaka, Zambia.

Ministry of Health and National AIDS Council, Zambia. 2008. Zambia Country Report, MultiSectoral AIDS Response Monitoring and Evaluation Biennial Report, 2006-2007. Lusaka, Zambia.

National AIDS Council. 2006. National HIV and AIDS Strategic Framework. Republic of Zambia, May 2006.

Newell, Marie-Louise, Heena Brahmbhatt, and Peter D. Ghys. 2004. Child mortality and HIV infection in Africa: a review. AIDS, 18(supplement 2): S27-S34 
Ngashi, N., C. Luo, D. Mulenga, and R. Gass. 2006. Innovative bottleneck-solving strategies for taking PMCT programs to scale: Learning from proven country experiences. The XVI International AIDS Conference, Toronto, Canada.

PEPFAR. 2005. Engendering Bold Leadership: The President's Emergency Plan for AIDS Relief, First Annual Report to Congress. Office of the United States Global AIDS Coordinator, Washington, DC.

PEPFAR. 2006. Action Today, A Foundation for Tomorrow: The President's Emergency Plan for AIDS Relief, Second Annual Report to Congress. Office of the United States Global AIDS Coordinator, Washington, DC.

PEPFAR. 2007. The Power of Partnerships: The President's Emergency Plan for AIDS Relief, Third Annual Report to Congress. Office of the United States Global AIDS Coordinator, Washington, DC.

Pop-Eleches, Cristian. 2006. The impact of an abortion ban on socioeconomic outcomes of children: Evidence from Romania. Journal of Political Economy, 114(4): 744-773.

Pop-Eleches, Cristian. 2010. The supply of birth control methods, education, and fertility: Evidence from Romania. Journal of Human Resources, 45(September): 971-997.

Pörtner, Claus. 2010. Sex selective abortions, fertility, and birth spacing. University of Washington working paper.

Preston, Samuel. 1975. Health programs and population growth. Population and Development Review, 1(2): 189-199.

Rutenberg, Naomi, Scott Geibel, Sam Kalibala, Chipepo Kankasa, Ruth Nduati, Dorothy Mbori Ngacha, Jennifer Oyieke, and Margaret Siwale. 2003. "Infant Feeding Counseling within Kenyan and Zambian PMTCT Services: How Well Does it Promote Good Feeding Practices?" Horizons Research Summary. Population Council: Washington, DC.

Sah, Raaj. 1991. The effects of child mortality changes on fertility choice and parental welfare. Journal of Political Economy, 99(3): 582-606.

Schmidt, Lucie. 2007. Effects of infertility insurance mandates on fertility. Journal of Health Economics 26(3): 431-446.

Schultz, T. Paul. 1997. Demand for children in low income countries. Chapter 8 in Handbook of Population and Family Economics, M. R. Rosenzweig and O. Stark, eds.

Soares, Rodrigo. 2005. Mortality reductions, educational attainment, and fertility choice. American Economic Review, 95(3): 580-601.

Spira, Rosemary, Phillipe Lepgage, Phillipe Msellati, Phillipe Van d Perre, Valeraine Leroy, Arlette Simonon, Etienee Karita, and Francois Dabis. 1999. Natural history of HIV type 1 infection in children: A five-year prospective study in Rwanda. Pediatrics, 104: D1-D9.

Steckelenburg, J., S. Kyanamina, M. Mukelabai, I. Wolffers, and J. van Roosmalen. 2004. Waiting too long: Low use of maternal health services in Kalabo, Zambia. Tropical Medicine and International Health, 9(3): 390-398. 
Stringer, Elizabeth, Moses Sinkala, Jeffrey Stringer, Elizabeth Mzyece, Ida Makuka, Robert Goldenberg, Pascal Kwane, Martha Chilufya, and Sten Vermund. 2003. Prevention of mother-to-child transmission of HIV in Africa: Successes and challenges in scaling-up a nevirapine-based program in Lusaka, Zambia. AIDS, 17(9): 1377-1382.

Stringer, Jeffrey, Moses Sinkala, Courtney Maclean, Jens Levy, Chipepo Kankasa, Alain DeGroot, Elizabeth Stringer, Edward Acosta, Robert Goldenberg, and Sten Vermund. 2005. Effectiveness of a city-wide program to prevent mother-to-child HIV transmission in Lusaka, Zambia. AIDS, 19(12): 1309-1315.

Taha, Taha, Newton Kumwenda, Robin Broadhead, Donald Hoover, Diane Markakis, Len van der Hoeven, George Liomba, John Chiphangwi, and Paolo Miotti. 1999. Mortality after the first year of life among human immunodeficiency virus type 1-infected and uninfected children. Pediatric Infectious Disease Journal, 18: 689-94.

Thornton, Rebecca. 2008. The demand for, and impact of, learning HIV status. American Economic Review, 98(5), 1829-1863.

Torpey, Kwasi, Mushota Kabaso, Prisca Kasonde, Rebecca Dirks, Maxmillian Bweupe, Catherine Thompson, and Ya Diul Mukadi. 2010. Increasing the uptake of prevention of mother-to-child transmission of HIV services in a resource-limited setting. BMC Health Services Research, 10:29.

UNAIDS. 2010. UNAIDS Report on the Global AIDS Epidemic. Joint United Nations Programme on HIV/AIDS.

UNICEF. 2010. Zambia: PMTCT Factsheet. United Nations Children's Fund.

UNICEF. 2012. Levels \& Trends in Child Mortality: Report 2012. UNICEF/WHO/The World Bank/United Nations.

Valente, Christine. 2011. Access to abortion, investments in neonatal health, and sex-selection: Evidence from Nepal. University of Sheffield working paper.

WHO. 2009. HIV and Infant Feeding: Revised Principles and Recommendations. World Health Organization.

WHO. 2010a. Toward Universal Access: Scaling up priority HIV/AIDS interventions in the health sector. World Health Organization.

WHO. 2010b. World Health Statistics 2010. World Health Organization.

Wilson, Nicholas. 2010. Antiretroviral therapy and demand for HIV testing: Evidence from Zambia. Williams College working paper.

Wilson, Nicholas. 2012. Prevention of mother-to-child transmission of HIV and reproductive behavior in Zambia. NBER Volume on African Economic Successes, University of Chicago Press, Sebastian Edwards, Simon Johnson, and David Weil, editors, forthcoming.

Wolpin, Kenneth. 1984. An estimable dynamic stochastic model of fertility and child mortality. Journal of Political Economy, 92(5): 852-874.

Young, Alwyn. 2005. The gift of the dying: The tragedy of AIDS and the welfare of future African generations. Quarterly Journal of Economics, 120(2): 423-466. 
Young, Alwyn. 2007. In sorrow to bring forth children. Journal of Economic Growth, 12: 283-327. 


\section{Figure 1: PMTCT Availability in Zambia, 2000-2007}

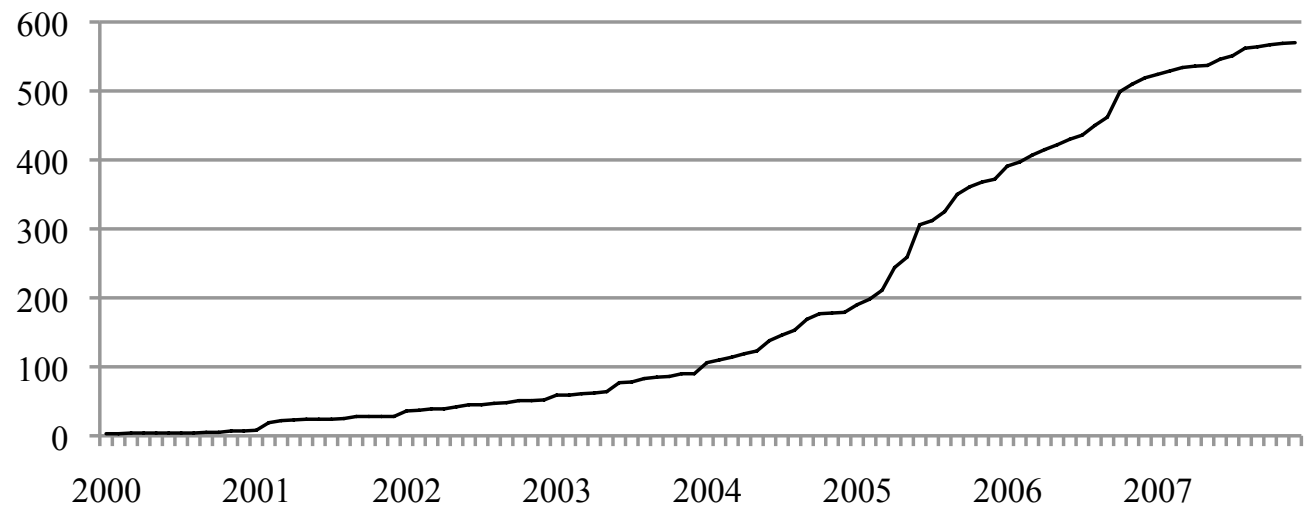

— cumulative number of PMTCT sites 


\section{Figure 2: PMTCT Sites by Year of Service Initiation}
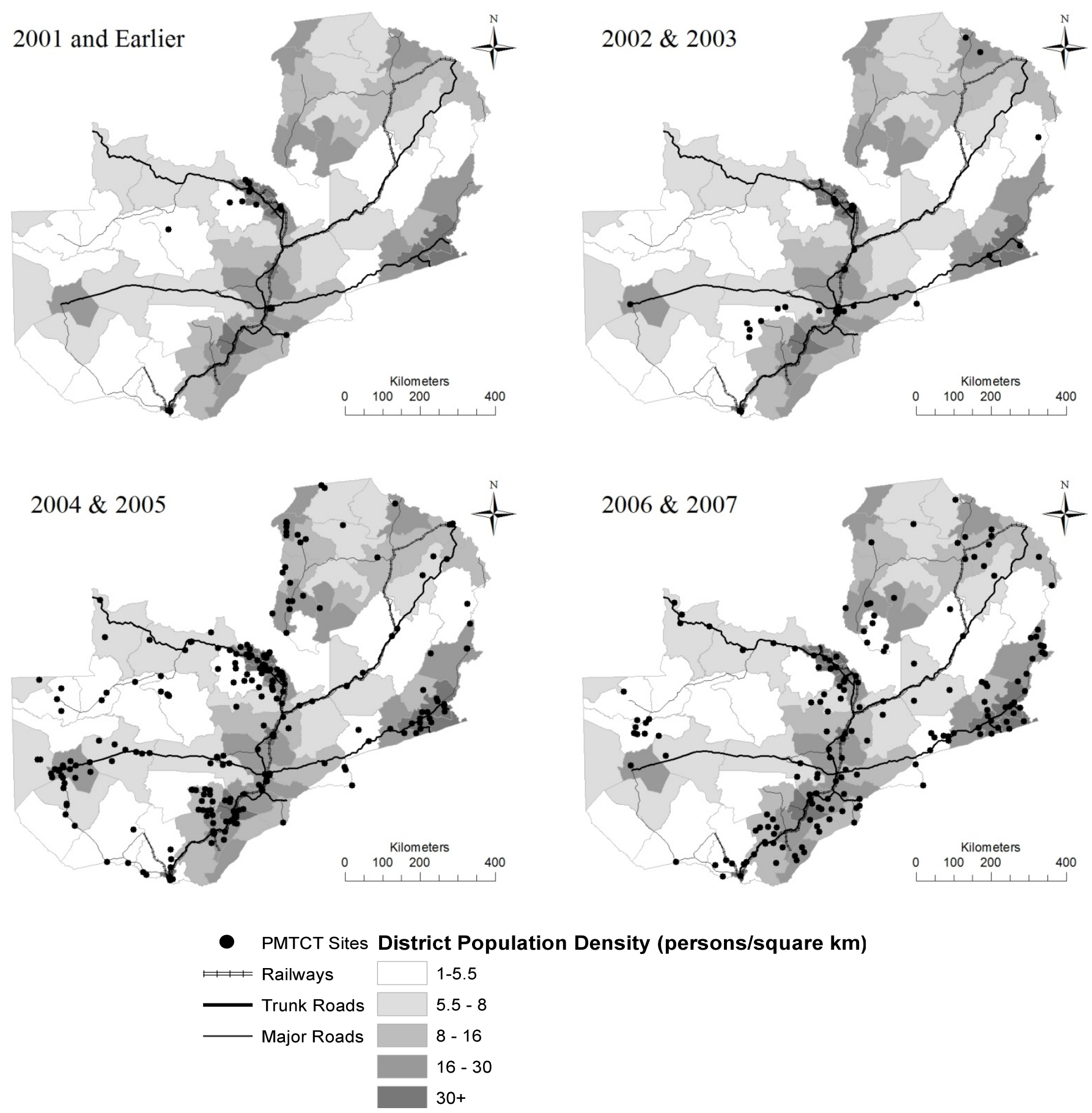

Notes: Data on the latitude and longitude of health facilities come from the 2006 Japanese International Cooperation Agency (JICA) Health Facilities Census (HFC). Data on the month and year PMTCT was introduced at a given facility come from the augmented 2006 JICA HFC. Data on transportation routes come from the Zambia Central Statistical Office (CSO). Data on district population levels come from the 2000 Zambia Census of Population and Housing. 
Figure 3: Semi-Parametric Difference-in-Differences Analysis of Effect of Local PMTCT on Pregnancy Rates

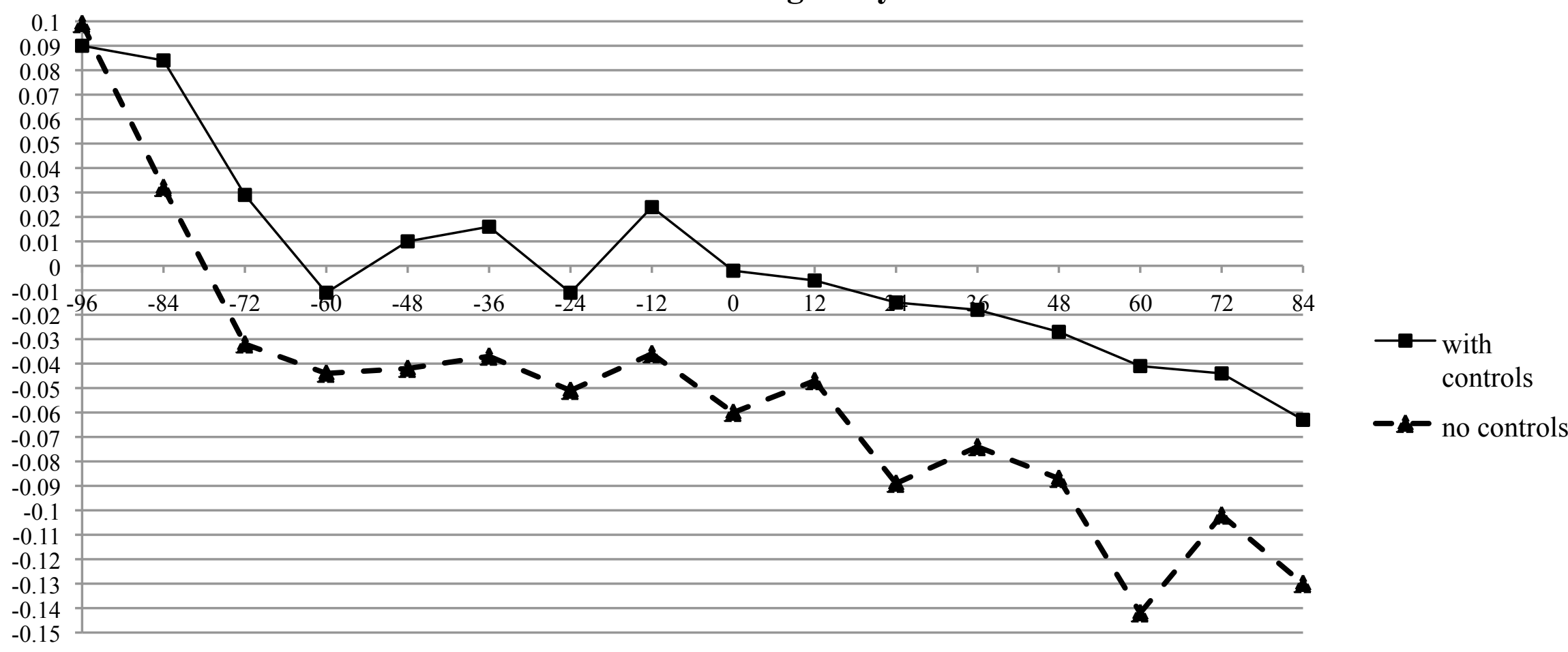


Table 1: Reproductive Behavior Among Female DHS and ZSBS Respondents

\begin{tabular}{|c|c|c|c|c|}
\hline & pregnant & $\begin{array}{c}\text { number of children } \\
\text { ever born }\end{array}$ & $\begin{array}{c}\text { currently } \\
\text { breastfeeding }\end{array}$ & $\begin{array}{c}\text { HIV } \\
\text { positive }\end{array}$ \\
\hline & (1) & $(2)$ & (3) & (4) \\
\hline \multicolumn{5}{|l|}{ Age } \\
\hline $15-19$ & 0.22 & 0.31 & 0.84 & 0.06 \\
\hline $20-24$ & 0.42 & 1.55 & 0.81 & 0.13 \\
\hline $25-29$ & 0.43 & 2.87 & 0.78 & 0.21 \\
\hline $30-34$ & 0.38 & 4.19 & 0.82 & 0.25 \\
\hline $35-39$ & 0.29 & 5.50 & 0.82 & 0.25 \\
\hline $40-44$ & 0.15 & 6.44 & 0.82 & 0.19 \\
\hline $45-49$ & 0.04 & 6.95 & 0.76 & 0.14 \\
\hline All females & 0.31 & 3.01 & 0.81 & 0.17 \\
\hline Observations & 17,820 & 17,805 & 5,507 & 5,698 \\
\hline
\end{tabular}

Notes: Pregnancy data come from the 2001 and 2007 DHS survey rounds and the 2003 and 2005 ZSBS survey rounds. Number of children ever born and breastfeeding data come from the 2001 and 2007 DHS survey rounds. HIV positive comes from HIV testing module in 2007 DHS. Pregnant is an indicator variable equal to one if the respondent reported being pregnant at any time in the past twelve months. Currently breastfeeding is a child level indicator variable equal to one a child age 24 months or younger is currently breastfeeding. HIV positive is an indicator variable equal to one if the respondent tested positive. 
Table 2: Characteristics of Female DHS and ZSBS Respondents by Proximity to PMTCT Site

\begin{tabular}{lcc}
\hline \hline & $\begin{array}{c}\text { within } 20 \mathrm{~km} \\
\text { of eventual PMTCT site }\end{array}$ & $\begin{array}{c}\text { greater than 20km } \\
\text { Sample: }\end{array}$ \\
\cline { 2 - 3 } & $(1)$ & from eventual PMTCT site \\
\hline
\end{tabular}

Panel A: Females age 15-49

Age

27.6

28.4

Completed primary

0.56

0.30

Completed secondary

0.11

0.02

Married

0.59

0.67

Any partner

0.73

0.78

Proportion unprotected sex

0.86

0.92

Pregnant

0.30

0.37

Number of children ever born

2.89

3.41

HIV positive

0.18

0.09

Piped water

0.42

0.03

Bed net

0.53

0.44

Observations

13,747

4,073

Panel B: Children age 0-24 months

Breastfeeding

0.80

0.84

Observations

3,996

1,511

Notes: Data come from the 2001 and 2007 DHS survey rounds and the 2003 and 2005 DHS survey rounds.

Completed primary and completed secondary are indicator variables for primary and secondary school completion, respectively. Data on sexual behavior come from the 2003 and 2005 DHS survey rounds. Any partner is an indicator variable for any sexual partner in past twelve months. Proportion unprotected sex is proportion of reported sex acts in a which a condom was used. HIV data come from the HIV testing module in the 2007 DHS. Pregnant is an indicator variable equal to one if the respondent reported being pregnant at any time in the past twelve months. HIV positive is an indicator variable equal to one if the respondent tested positive. Piped water and bed net are indicator variables for household acess to piped water and household bed net ownership, respectively. Currently breastfeeding is a child level indicator variable equal to one a child age 24 months or younger is currently breastfeeding. 
Table 3: Effect of Local PMTCT on Reproductive Behavior

\begin{tabular}{|c|c|c|c|c|c|c|}
\hline & $(1)$ & $(2)$ & (3) & $(4)$ & $(5)$ & $(6)$ \\
\hline \multicolumn{7}{|l|}{ Panel A: Pregnant } \\
\hline PMTCT within $20 \mathrm{~km}$ & $\begin{array}{c}-0.046^{* * *} \\
(0.011)\end{array}$ & $\begin{array}{c}-0.089^{* * *} \\
(0.012)\end{array}$ & $\begin{array}{c}-0.063 * * * \\
(0.014)\end{array}$ & $\begin{array}{c}-0.038^{* * *} \\
(0.010)\end{array}$ & $\begin{array}{c}-0.039 * * * \\
(0.013)\end{array}$ & $\begin{array}{l}-0.020 \\
(0.017)\end{array}$ \\
\hline Month times year fixed effects & NO & YES & YES & YES & YES & YES \\
\hline Control for PMTCT expansion stage & NO & NO & YES & YES & YES & YES \\
\hline Individual level controls & NO & NO & NO & YES & YES & YES \\
\hline Province fixed effects and linear trends & NO & NO & NO & NO & YES & YES \\
\hline $\begin{array}{l}\text { Controls for other HIV/AIDS services, } \\
\text { piped water, and bed net ownership }\end{array}$ & NO & NO & NO & NO & NO & YES \\
\hline Observations & 17,820 & 17,819 & 17,819 & 17,817 & 17,817 & 15,734 \\
\hline \multicolumn{7}{|l|}{ Panel B: Breastfeeding } \\
\hline PMTCT within $20 \mathrm{~km}$ & $\begin{array}{r}0.037^{* *} \\
(0.015)\end{array}$ & $\begin{array}{c}0.095 * * * \\
(0.018)\end{array}$ & $\begin{array}{c}0.141 * * * \\
(0.020)\end{array}$ & $\begin{array}{c}0.144 * * * \\
(0.020)\end{array}$ & $\begin{array}{c}0.211^{* * *} \\
(0.025)\end{array}$ & $\begin{array}{r}0.236 * * * \\
(0.026)\end{array}$ \\
\hline Month times year fixed effects & NO & YES & YES & YES & YES & YES \\
\hline Control for PMTCT expansion stage & NO & NO & YES & YES & YES & YES \\
\hline Individual level controls & NO & NO & NO & YES & YES & YES \\
\hline Province fixed effects and linear trends & NO & NO & NO & NO & YES & YES \\
\hline $\begin{array}{l}\text { Controls for other HIV/AIDS services, } \\
\text { piped water, and bed net ownership }\end{array}$ & NO & NO & NO & NO & NO & YES \\
\hline Observations & 5,507 & 5,507 & 5,507 & 5,507 & 5,507 & 5,507 \\
\hline
\end{tabular}

Notes: Pregnancy data come from the 2001 and 2007 DHS survey rounds and the 2003 and 2005 ZSBS survey rounds. Breastfeeding data come from the 2001 and 2007 DHS survey rounds. Pregnant is an indicator variable for being pregnant sometime in the twelve months prior to the survey date, inclusive. Breastfeeding is an indicator variable equal to one if the respondent is breastfeeding conditional on having a child age 0-24 months. "PMTCT within $20 \mathrm{~km}$ " is an indicator variable equal to one if a health clinic within 20 kilometers of the respondent offered PMTCT at least twelve months prior to the survey date. All specifications include an indicator variable for whether PMTCT was ever introduced within $20 \mathrm{~km}$ of the respondent. Parameters estimated using ordinary least squares (OLS) regressions. Standard errors are in parentheses and are clustered by Standard Enumeration Area (SEA). *** Significant at the 1 percent level, ** Significant at the 5 percent level, * Significant at the 10 percent level. 
Table 4: Spatial Heterogeneity in Effect of Local PMTCT on Reproductive Behavior

\begin{tabular}{|c|c|c|c|c|c|c|}
\hline & $(1)$ & $(2)$ & (3) & $(4)$ & $(5)$ & $(6)$ \\
\hline \multicolumn{7}{|l|}{ Panel A: Pregnant } \\
\hline PMTCT within $10 \mathrm{~km}$ & $\begin{array}{l}-0.049 * * * \\
(0.017)\end{array}$ & $\begin{array}{c}-0.064 * * * \\
(0.017)\end{array}$ & $\begin{array}{l}-0.061 * * * \\
(0.017)\end{array}$ & $\begin{array}{l}-0.021 \\
(0.014)\end{array}$ & $\begin{array}{c}-0.028 * * \\
(0.014)\end{array}$ & $\begin{array}{l}-0.021 \\
(0.015)\end{array}$ \\
\hline PMTCT within $20 \mathrm{~km}$ & $\begin{array}{c}0.000 \\
(0.029)\end{array}$ & $\begin{array}{l}-0.001 \\
(0.029)\end{array}$ & $\begin{array}{c}0.009 \\
(0.028)\end{array}$ & $\begin{array}{l}-0.012 \\
(0.021)\end{array}$ & $\begin{array}{l}-0.027 \\
(0.021)\end{array}$ & $\begin{array}{l}-0.013 \\
(0.022)\end{array}$ \\
\hline PMTCT within $30 \mathrm{~km}$ & $\begin{array}{c}0.000 \\
(0.026)\end{array}$ & $\begin{array}{l}-0.040 \\
(0.027)\end{array}$ & $\begin{array}{l}-0.031 \\
(0.027)\end{array}$ & $\begin{array}{l}-0.014 \\
(0.019)\end{array}$ & $\begin{array}{c}0.008 \\
(0.020)\end{array}$ & $\begin{array}{c}0.007 \\
(0.019)\end{array}$ \\
\hline Month times year fixed effects & NO & YES & YES & YES & YES & YES \\
\hline Control for PMTCT expansion stage & NO & NO & YES & YES & YES & YES \\
\hline Individual level controls & NO & NO & NO & YES & YES & YES \\
\hline Province fixed effects and linear trends & NO & NO & NO & NO & YES & YES \\
\hline $\begin{array}{l}\text { Controls for other HIV/AIDS services, } \\
\text { piped water, and bed net ownership }\end{array}$ & NO & NO & NO & NO & NO & YES \\
\hline Observations & 17,820 & 17,819 & 17,819 & 17,817 & 17,817 & 15,734 \\
\hline \multicolumn{7}{|l|}{ Panel B: Breastfeeding } \\
\hline PMTCT within $10 \mathrm{~km}$ & $\begin{array}{l}-0.011 \\
(0.027)\end{array}$ & $\begin{array}{l}0.005 \\
(0.027)\end{array}$ & $\begin{array}{c}0.005 \\
(0.026)\end{array}$ & $\begin{array}{c}0.010 \\
(0.025)\end{array}$ & $\begin{array}{l}0.013 \\
(0.024)\end{array}$ & $\begin{array}{c}0.024 \\
(0.025)\end{array}$ \\
\hline PMTCT within $20 \mathrm{~km}$ & $\begin{array}{l}0.051^{* *} \\
(0.022)\end{array}$ & $\begin{array}{c}0.103 * * * \\
(0.023)\end{array}$ & $\begin{array}{c}0.143 * * * \\
(0.024)\end{array}$ & $\begin{array}{c}0.141 * * * \\
(0.024)\end{array}$ & $\begin{array}{c}0.206 * * * \\
(0.026)\end{array}$ & $\begin{array}{c}0.223 * * * \\
(0.027)\end{array}$ \\
\hline PMTCT within $30 \mathrm{~km}$ & $\begin{array}{l}-0.009 \\
(0.037)\end{array}$ & $\begin{array}{c}0.011 \\
(0.034)\end{array}$ & $\begin{array}{c}0.014 \\
(0.035)\end{array}$ & $\begin{array}{c}0.012 \\
(0.034)\end{array}$ & $\begin{array}{l}-0.006 \\
(0.034)\end{array}$ & $\begin{array}{l}-0.012 \\
(0.030)\end{array}$ \\
\hline Month times year fixed effects & NO & YES & YES & YES & YES & YES \\
\hline Control for PMTCT expansion stage & NO & NO & YES & YES & YES & YES \\
\hline Individual level controls & NO & NO & NO & YES & YES & YES \\
\hline Province fixed effects and linear trends & NO & NO & NO & NO & YES & YES \\
\hline $\begin{array}{l}\text { Controls for other HIV/AIDS services, } \\
\text { piped water, and bed net ownership }\end{array}$ & NO & NO & NO & NO & NO & YES \\
\hline Observations & 5,507 & 5,507 & 5,507 & 5,507 & 5,507 & 5,507 \\
\hline
\end{tabular}

Notes: Pregnancy data come from the 2001 and 2007 DHS survey rounds and the 2003 and 2005 ZSBS survey rounds. Breastfeeding data come from the 2001 and 2007 DHS survey rounds. Pregnant is an indicator variable for being pregnant sometime in the twelve months prior to the survey date, inclusive. Breastfeeding is an indicator variable equal to one if the respondent is breastfeeding conditional on having a child age 0-24 months. "PMTCT within $20 \mathrm{~km}$ " is an indicator variable equal to one if a health clinic within 20 kilometers of the respondent offered PMTCT at least twelve months prior to the survey date. "PMTCT within $10 \mathrm{~km}$ " and "PMTCT within $30 \mathrm{~km}$ " are defined similarly. All specifications include an indicator variable for whether PMTCT was ever introduced within $20 \mathrm{~km}$ of the respondent. Parameters estimated using ordinary least squares (OLS) regression. Standard errors are in parentheses and are clustered by Standard Enumeration Area (SEA).

*** Significant at the 1 percent level, ** Significant at the 5 percent level, * Significant at the 10 percent level. 
Table 5: Dynamic Effects of Local PMTCT on Reproductive Behavior

\begin{tabular}{|c|c|c|c|c|c|c|}
\hline & $(1)$ & $(2)$ & (3) & $(4)$ & $(5)$ & $(6)$ \\
\hline \multicolumn{7}{|l|}{ Panel A: Pregnant } \\
\hline PMTCT within $20 \mathrm{~km}$ & $\begin{array}{c}-0.030^{* *} \\
(0.013)\end{array}$ & $\begin{array}{l}-0.072 * * * \\
(0.013)\end{array}$ & $\begin{array}{l}-0.058^{* * *} \\
(0.014)\end{array}$ & $\begin{array}{c}-0.035^{* * *} \\
(0.011)\end{array}$ & $\begin{array}{l}-0.036^{* * *} \\
(0.013)\end{array}$ & $\begin{array}{l}-0.018 \\
(0.016)\end{array}$ \\
\hline PMTCT within $20 \mathrm{~km}$ at least 36 months & $\begin{array}{c}-0.035^{* *} \\
(0.014)\end{array}$ & $\begin{array}{c}-0.044 * * * \\
(0.014)\end{array}$ & $\begin{array}{l}-0.024^{*} \\
(0.014)\end{array}$ & $\begin{array}{l}-0.017 \\
(0.011)\end{array}$ & $\begin{array}{l}-0.021 \\
(0.013)\end{array}$ & $\begin{array}{l}-0.018 \\
(0.015)\end{array}$ \\
\hline $\mathrm{P}>\mathrm{F}(\mathrm{PMTCT}+\mathrm{PMTCT}$ at least thirty-six months $=0)$ & 0.000 & 0.000 & 0.000 & 0.000 & 0.001 & 0.086 \\
\hline Month times year fixed effects & NO & YES & YES & YES & YES & YES \\
\hline Control for PMTCT expansion stage & $\mathrm{NO}$ & NO & YES & YES & YES & YES \\
\hline Individual level controls & $\mathrm{NO}$ & NO & NO & YES & YES & YES \\
\hline Province fixed effects and linear trends & NO & NO & NO & NO & YES & YES \\
\hline $\begin{array}{l}\text { Controls for other HIV/AIDS services, } \\
\text { piped water, and bed net ownership }\end{array}$ & NO & NO & NO & NO & NO & YES \\
\hline Observations & 17,820 & 17,819 & 17,819 & 17,817 & 17,817 & 15,736 \\
\hline \multicolumn{7}{|l|}{ Panel B: Breastfeeding } \\
\hline PMTCT within $20 \mathrm{~km}$ & $\begin{array}{c}0.051^{* * *} \\
(0.018)\end{array}$ & $\begin{array}{c}0.106^{* * *} \\
(0.019)\end{array}$ & $\begin{array}{c}0.140^{* * *} \\
(0.020)\end{array}$ & $\begin{array}{c}0.144 * * * \\
(0.020)\end{array}$ & $\begin{array}{c}0.209^{* * *} \\
(0.024)\end{array}$ & $\begin{array}{c}0.233 * * * \\
(0.025)\end{array}$ \\
\hline PMTCT within $20 \mathrm{~km}$ at least 36 months & $\begin{array}{l}-0.037 \\
(0.023)\end{array}$ & $\begin{array}{l}-0.032 \\
(0.024)\end{array}$ & $\begin{array}{c}0.001 \\
(0.025)\end{array}$ & $\begin{array}{c}0.001 \\
(0.025)\end{array}$ & $\begin{array}{c}0.015 \\
(0.034)\end{array}$ & $\begin{array}{c}0.030 \\
(0.036)\end{array}$ \\
\hline $\mathrm{P}>\mathrm{F}(\mathrm{PMTCT}+\mathrm{PMTCT}$ at least thirty-six months $=0)$ & 0.498 & 0.003 & 0.000 & 0.000 & 0.000 & 0.000 \\
\hline Month times year fixed effects & NO & YES & YES & YES & YES & YES \\
\hline Control for PMTCT expansion stage & NO & NO & YES & YES & YES & YES \\
\hline Individual level controls & NO & NO & NO & YES & YES & YES \\
\hline Province fixed effects and linear trends & NO & NO & NO & NO & YES & YES \\
\hline $\begin{array}{l}\text { Controls for other HIV/AIDS services, } \\
\text { piped water, and bed net ownership }\end{array}$ & NO & NO & NO & NO & NO & YES \\
\hline Observations & 5,507 & 5,507 & 5,507 & 5,507 & 5,507 & 5,507 \\
\hline
\end{tabular}

Notes: Pregnancy data come from the 2001 and 2007 DHS survey rounds and the 2003 and 2005 ZSBS survey rounds. Breastfeeding data come from the 2001 and 2007 DHS survey rounds. Pregnant is an indicator variable for being pregnant sometime in the twelve months prior to the survey date, inclusive. Breastfeeding is an indicator variable equal to one if the respondent is breastfeeding conditional on having a child age $0-24$ months. "PMTCT within $20 \mathrm{~km}$ " is an indicator variable equal to one if a health clinic within 20 kilometers of the respondent offered PMTCT at least twelve months prior to the survey date. "PMTCT within $20 \mathrm{~km}$ at least 36 months" is an indicator variable equal to one if a health clinic within 20 kilometers of the respondent offered PMTCT at least 36 months prior to the survey date. All specifications include an indicator variable for whether PMTCT was ever introduced within $20 \mathrm{~km}$ of the respondent. Parameters estimated using ordinary least squares (OLS) regression. Standard errors are in parentheses and are clustered by Standard Enumeration Area (SEA).

*** Significant at the 1 percent level, ** Significant at the 5 percent level, * Significant at the 10 percent level. 
Table 6: Heterogeneity by HIV Prevalence in Effect of Local PMTCT on Pregnancy

\begin{tabular}{|c|c|c|c|c|c|c|}
\hline & $(1)$ & $(2)$ & (3) & (4) & $(\overline{c(5)}$ & (6) \\
\hline \multicolumn{7}{|l|}{ Panel A: Continuous measure of HIV prevalence } \\
\hline PMTCT within $20 \mathrm{~km}$ & $\begin{array}{c}-0.039^{* *} \\
(0.018)\end{array}$ & $\begin{array}{l}-0.089^{* * *} \\
(0.019)\end{array}$ & $\begin{array}{c}-0.063^{* * *} \\
(0.021)\end{array}$ & $\begin{array}{l}-0.011 \\
(0.016)\end{array}$ & $\begin{array}{l}-0.023 \\
(0.017)\end{array}$ & $\begin{array}{l}-0.006 \\
(0.020)\end{array}$ \\
\hline PMTCT within $20 \mathrm{~km} *$ HIV prevalence & $\begin{array}{l}-0.063 \\
(0.086)\end{array}$ & $\begin{array}{l}-0.031 \\
(0.084)\end{array}$ & $\begin{array}{l}-0.025 \\
(0.084)\end{array}$ & $\begin{array}{c}-0.149 * * \\
(0.069)\end{array}$ & $\begin{array}{c}-0.092 \\
(0.076)\end{array}$ & $\begin{array}{c}-0.078 \\
(0.082)\end{array}$ \\
\hline $\mathrm{P}>\mathrm{F}\left(\mathrm{PMTCT}+\mathrm{PMTCT}^{*} \mathrm{HIV}\right.$ prevalence $\left.=0\right)$ & 0.152 & 0.087 & 0.210 & 0.006 & 0.079 & 0.253 \\
\hline Month times year fixed effects & NO & YES & YES & YES & YES & YES \\
\hline Control for PMTCT expansion stage & NO & NO & YES & YES & YES & YES \\
\hline Individual level controls & NO & NO & NO & YES & YES & YES \\
\hline Province fixed effects and linear trends & NO & NO & NO & NO & YES & YES \\
\hline $\begin{array}{l}\text { Controls for other HIV/AIDS services, } \\
\text { piped water, and bed net ownership }\end{array}$ & NO & NO & NO & NO & NO & YES \\
\hline Observations & 17,818 & 17,817 & 17,817 & 17,817 & 17,817 & 15,736 \\
\hline \multicolumn{7}{|l|}{ Panel B: Binary measure of HIV prevalence } \\
\hline PMTCT within $20 \mathrm{~km}$ & $\begin{array}{l}-0.052^{* * *} \\
(0.014)\end{array}$ & $\begin{array}{l}-0.101 * * * \\
(0.015)\end{array}$ & $\begin{array}{l}-0.080^{* * *} \\
(0.016)\end{array}$ & $\begin{array}{l}-0.021^{*} \\
(0.012)\end{array}$ & $\begin{array}{l}-0.025^{*} \\
(0.013)\end{array}$ & $\begin{array}{l}-0.004 \\
(0.018)\end{array}$ \\
\hline PMTCT within $20 \mathrm{~km} *$ HIV prevalence at median or above & $\begin{array}{c}0.009 \\
(0.016)\end{array}$ & $\begin{array}{c}0.017 \\
(0.016)\end{array}$ & $\begin{array}{c}0.021 \\
(0.016)\end{array}$ & $\begin{array}{c}-0.032 * * \\
(0.013)\end{array}$ & $\begin{array}{l}-0.027^{*} \\
(0.014)\end{array}$ & $\begin{array}{l}-0.025^{*} \\
(0.015)\end{array}$ \\
\hline $\mathrm{P}>\mathrm{F}\left(\mathrm{PMTCT}+\mathrm{PMTCT}^{*} \mathrm{HIV}\right.$ prev median above $\left.=0\right)$ & 0.001 & 0.000 & 0.000 & 0.000 & 0.000 & 0.114 \\
\hline Month times year fixed effects & NO & YES & YES & YES & YES & YES \\
\hline Control for PMTCT expansion stage & NO & NO & YES & YES & YES & YES \\
\hline Individual level controls & NO & NO & NO & YES & YES & YES \\
\hline Province fixed effects and linear trends & NO & NO & NO & NO & YES & YES \\
\hline $\begin{array}{l}\text { Controls for other HIV/AIDS services, } \\
\text { piped water, and bed net ownership }\end{array}$ & NO & NO & NO & NO & NO & YES \\
\hline Observations & 17,818 & 17,818 & 17,817 & 17,817 & 17,817 & 15,734 \\
\hline
\end{tabular}

Notes: Pregnancy data come from the 2001 and 2007 DHS survey rounds and the 2003 and 2005 ZSBS survey rounds. Pregnant is an indicator variable for being pregnant sometime in the twelve months prior to the survey date, inclusive. "PMTCT within $20 \mathrm{~km} "$ is an indicator variable equal to one if a health clinic within 20 kilometers of the respondent offered PMTCT at least twelve months prior to the survey date. "HIV prevalence" is measrued as the HIV prevalence in the respondent's demographci group, defined as the interaction of gender, five year age group, and province of residence. "HIV prevalence at median or above" is an indicator variable equal to one if HIV prevalence in the respondent's demographic group is equal to or greater than median HIV prevalence in Zambia. All specifications include an indicator variable for whether PMTCT was ever introduced within $20 \mathrm{~km}$ of the respondent. Parameters estimated using ordinary least squares (OLS) regression. Standard errors are in parentheses and are clustered by Standard Enumeration Area (SEA).

*** Significant at the 1 percent level, $* *$ Significant at the 5 percent level, * Significant at the 10 percent level. 
Table 7: Heterogeneity by HIV Prevalence in Effect of Local PMTCT on Breastfeeding

\begin{tabular}{|c|c|c|c|c|c|c|}
\hline & $(1)$ & $(2)$ & $(3)$ & 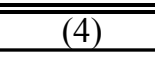 & 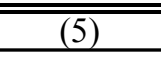 & $(6)$ \\
\hline \multicolumn{7}{|l|}{ Panel A: Continuous measure of HIV prevalence } \\
\hline PMTCT within $20 \mathrm{~km}$ & $\begin{array}{c}0.031 \\
(0.028)\end{array}$ & $\begin{array}{c}0.096 * * * \\
(0.030)\end{array}$ & $\begin{array}{c}0.139^{* * *} \\
(0.030)\end{array}$ & $\begin{array}{c}0.154 * * * \\
(0.030)\end{array}$ & $\begin{array}{l}0.215^{* * *} \\
(0.037)\end{array}$ & $\begin{array}{c}0.238^{* * *} \\
(0.038)\end{array}$ \\
\hline PMTCT within $20 \mathrm{~km} *$ HIV prevalence & $\begin{array}{l}0.058 \\
(0.137)\end{array}$ & $\begin{array}{c}0.02 \\
(0.137)\end{array}$ & $\begin{array}{c}0.015 \\
(0.135)\end{array}$ & $\begin{array}{l}-0.048 \\
(0.136)\end{array}$ & $\begin{array}{l}-0.023 \\
(0.155)\end{array}$ & $\begin{array}{l}-0.012 \\
(0.155)\end{array}$ \\
\hline $\mathrm{P}>\mathrm{F}\left(\mathrm{PMTCT}+\mathrm{PMTCT}^{*} \mathrm{HIV}\right.$ prevalence $\left.=0\right)$ & 0.437 & 0.315 & 0.179 & 0.359 & 0.142 & 0.081 \\
\hline Month times year fixed effects & NO & YES & YES & YES & YES & YES \\
\hline Control for PMTCT expansion stage & NO & NO & YES & YES & YES & YES \\
\hline Individual level controls & NO & NO & NO & YES & YES & YES \\
\hline Province fixed effects and linear trends & NO & NO & NO & NO & YES & YES \\
\hline $\begin{array}{l}\text { Controls for other HIV/AIDS services, } \\
\text { piped water, and bed net ownership }\end{array}$ & NO & NO & NO & NO & NO & YES \\
\hline Observations & 5,507 & 5,507 & 5,507 & 5,507 & 5,507 & 5,507 \\
\hline \multicolumn{7}{|l|}{ Panel B: Binary measure of HIV prevalence } \\
\hline PMTCT within $20 \mathrm{~km}$ & $\begin{array}{r}0.047^{* *} \\
(0.021)\end{array}$ & $\begin{array}{c}0.108^{* * *} \\
(0.023)\end{array}$ & $\begin{array}{c}0.149^{* * *} \\
(0.023)\end{array}$ & $\begin{array}{c}0.153^{* * *} \\
(0.023)\end{array}$ & $\begin{array}{c}0.217 * * * \\
(0.028)\end{array}$ & $\begin{array}{c}0.242^{* * *} \\
(0.030)\end{array}$ \\
\hline PMTCT within $20 \mathrm{~km} *$ HIV prevalence at median or above & $\begin{array}{l}-0.011 \\
(0.026)\end{array}$ & $\begin{array}{l}-0.016 \\
(0.026)\end{array}$ & $\begin{array}{l}-0.014 \\
(0.026)\end{array}$ & $\begin{array}{l}-0.015 \\
(0.026)\end{array}$ & $\begin{array}{l}-0.009 \\
(0.028)\end{array}$ & $\begin{array}{l}-0.009 \\
(0.027)\end{array}$ \\
\hline $\mathrm{P}>\mathrm{F}(\mathrm{PMTCT}+\mathrm{PMTCT} *$ HIV prev median above $=0)$ & 0.065 & 0.000 & 0.000 & 0.000 & 0.000 & 0.000 \\
\hline Month times year fixed effects & NO & YES & YES & YES & YES & YES \\
\hline Control for PMTCT expansion stage & NO & NO & YES & YES & YES & YES \\
\hline Individual level controls & NO & NO & NO & YES & YES & YES \\
\hline Province fixed effects and linear trends & NO & NO & NO & NO & YES & YES \\
\hline $\begin{array}{l}\text { Controls for other HIV/AIDS services, } \\
\text { piped water, and bed net ownership }\end{array}$ & NO & NO & NO & NO & NO & YES \\
\hline Observations & 5,507 & 5,507 & 5,507 & 5,507 & 5,507 & 5,507 \\
\hline
\end{tabular}

Notes: Breastfeeding data come from the 2001 and 2007 DHS survey rounds. Breastfeeding is an indicator variable equal to one if the respondent is breastfeeding conditional on having a child age 0-24 months. "PMTCT within $20 \mathrm{~km}$ " is an indicator variable equal to one if a health clinic within 20 kilometers of the respondent offered PMTCT at least twelve months prior to the survey date. "HIV prevalence" is measrued as the HIV prevalence in the respondent's demographci group, defined as the interaction of gender, five year age group, and province of residence. "HIV prevalence at median or above" is an indicator variable equal to one if HIV prevalence in the respondent's demographic group is equal to or greater than median HIV prevalence in Zambia. All specifications include an indicator variable for whether PMTCT was ever introduced within $20 \mathrm{~km}$ of the respondent. Parameters estimated using ordinary least squares (OLS) regression. Standard errors are in parentheses and are clustered by Standard Enumeration Area (SEA).

*** Significant at the 1 percent level, ** Significant at the 5 percent level, * Significant at the 10 percent level. 